\title{
Magnetic anisotropy in spin-3/2 with heavy ligand in honeycomb Mott insulators: Application to $\mathrm{CrI}_{3}$
}

\author{
P. Peter Stavropoulos $\odot,{ }^{1}$ Xiaoyu Liu, ${ }^{1}$ and Hae-Young Kee $\circledast^{1,2, *}$ \\ ${ }^{1}$ Department of Physics and Center for Quantum Materials, University of Toronto, 60 St. George Street, Toronto, Ontario, Canada M5S 1A7 \\ ${ }^{2}$ Canadian Institute for Advanced Research, Toronto, Ontario, Canada M5G $1 Z 8$
}

(Received 10 September 2020; revised 23 December 2020; accepted 22 January 2021; published 8 March 2021)

\begin{abstract}
Ferromagnetism in two-dimensional $\mathrm{CrI}_{3}$ has generated a lot of excitement, and it was recently proposed that the spin-orbit coupling (SOC) in iodine may generate bond-dependent spin interactions leading to magnetic anisotropy. Here, we derive a microscopic spin model of $S=3 / 2$ on transition metals surrounded by heavy ligands in honeycomb Mott insulators using a strong-coupling perturbation theory. For ideal octahedra we find Heisenberg and Kitaev interactions, which favor the magnetic moment along the cubic axis via quantum fluctuations. When a slight trigonal distortion of the octahedra is present together with the SOC, three additional interactions arise, composed of the off-diagonal symmetric $\Gamma$ and $\Gamma^{\prime}$ and single-ion anisotropy. The resulting magnetic anisotropy pins the moment perpendicular to the honeycomb plane as observed in a single layer of $\mathrm{CrI}_{3}$, suggesting the significance of SOC and trigonal distortion in understanding magnetism of two-dimensional Mott insulators. A comparison with the spin-orbit coupled $J_{\text {eff }}=1 / 2$ and $S=1$ models is also presented.
\end{abstract}

DOI: 10.1103/PhysRevResearch.3.013216

\section{INTRODUCTION}

Transition metal trihalides (TMTs) are layered materials composed of transition metals (M) and halides (X) of group 9 in a 1:3 ratio. They have a honeycomb layered structure, and depending on the filling of the $d$ orbitals in the transition metals, some are semiconductors and some are metals [1]. Among them, $\mathrm{RuCl}_{3}, \mathrm{VI}_{3}$, and $\mathrm{CrI}_{3}$ are Mott insulators. Magnetic orderings in these systems further establish the importance of electronic correlations and call for a microscopic understanding of spin models. For example, based on a strong-coupling perturbation theory of the generic spin model [2-4], it was shown that $\alpha-\mathrm{RuCl}_{3}$ described by the effective spin $J_{\text {eff }}=1 / 2$ has dominant bond-dependent Kitaev and off-diagonal symmetric $\Gamma$ interactions [5,6]. $\mathrm{RuCl}_{3}$ has become an emergent candidate for the spin-1/2 Kitaev spin liquid [7]. Intense research activities on various properties of $\mathrm{RuCl}_{3}$ have been carried out [6,8-14], and recently a magnetic-field-induced spin liquid was suggested [15-21].

In parallel, theoretical interest in the ground state of higher-spin Kitaev models was initiated by classical model studies [22,23]. The classical Kitaev model has a macroscopic degeneracy named a classical spin liquid [22], but the higherspin quantum Kitaev model is not exactly solvable, and the ground state is currently unknown. Various numerical studies such as exact diagonalization on $S=1$ suggested that the

\footnotetext{
*hykee@physics.utoronto.ca

Published by the American Physical Society under the terms of the Creative Commons Attribution 4.0 International license. Further distribution of this work must maintain attribution to the author(s) and the published article's title, journal citation, and DOI.
}

ground state is possibly a spin liquid with gapless excitations [24]. These studies were mainly of theoretical interest, until a microscopic derivation of the $S=1$ Kitaev-Heisenberg model in multiorbital systems was found [25]. Heavy ligand spin-orbit coupling (SOC) and strong Hund's coupling in $e_{\mathrm{g}}$ orbitals are a way to generate $S=1$ bond-dependent Kitaev interaction. The magnetic field effects on the $S=1$ Kitaev model have also been investigated [26-28].

The bond-dependent interactions have recently been adopted into TMT systems, because the nearest-neighbor (NN) Heisenberg $J$, Kitaev $K$, and $\Gamma$ interactions are allowed based on the symmetry of the lattice $[4,29,30]$. In particular, ferromagnetism in a single-layer $\mathrm{CrI}_{3}$ has generated excitement in recent years [31-45]. $\mathrm{CrI}_{3}$ is a ferromagnetic (FM) insulator with $T_{c} \sim 61 \mathrm{~K}$ for bulk samples [46-48]. Singlelayer $\mathrm{CrI}_{3}$ was successfully synthesized, which showed an FM ordering with $T_{c} \sim 45 \mathrm{~K}$ [49]. The two-dimensional FM Heisenberg model is insufficient to explain finite $T_{c}$, i.e., the Mermin-Wagner theorem [50], and several theoretical models were proposed to explain the magnetic anisotropy. They include the XXZ model [51,52], single-ion anisotropy and Kitaev interactions [53], and large Kitaev and small symmetric off-diagonal $\Gamma$ interaction [54].

While the Heisenberg, Kitaev, and $\Gamma$ interactions are allowed by the symmetry, and found to be significant in the earlier derivations for lower spins [3,4,25], their strengths may not be significant in $S=3 / 2$ systems. Thus a microscopic derivation of a $S=3 / 2$ model is necessary to find the sources of the magnetic anisotropy. Here, we derive a NN spin model for $S=3 / 2$ with three electrons in $t_{2 \mathrm{~g}}$ orbitals of transition metal sites and strong SOC in $p$ orbitals of ligands. We take into account strong electron-electron interactions in multiorbital systems including Hund's coupling and effects of trigonal distortions present in the $R \overline{3}$ rhombohedral lattice. 
Contributions from $e_{\mathrm{g}}$ orbitals are important as shown below. The minimal NN model includes $J, K, \Gamma$, another symmetric off-diagonal $\Gamma^{\prime}$ [55], and single-ion anisotropy $A_{c}$ along the $\hat{c}$ axis, denoted as the $J-K-\Gamma-\Gamma^{\prime}-A_{c}$ model.

The rest of the paper is organized as follows. In Sec. II, the on-site Kanamori interaction and tight binding Hamiltonian are presented. In Sec. III, we derive the NN spin model consisting of Kitaev and Heisenberg interactions for the ideal octahedra environment using standard perturbation theory. In Sec. IV, we study the spin model with trigonal distortion present in the $R \overline{3}$ rhombohedral lattice. This includes the distortion-induced hopping matrix elements and three additional spin interactions generated via combined effects of SOC and distortion. In Sec. V, we apply the theory to $\mathrm{CrI}_{3}$ and present the exchange interaction strengths using tight binding parameter sets obtained by density functional theory. The effects of the resulting magnetic anisotropy on the moment direction are found in Sec. VI. In Sec. VII we discuss the origin of the spin gap, finite $T_{c}$, and spin wave spectrum within the $J-K-\Gamma-\Gamma^{\prime}-A_{c}$ model. The effects of the the second-NN Dzyaloshinskii-Moriya (DM) interaction are also discussed. Finally, in Sec. VIII, we summarize our results and compare with $J_{\text {eff }}=1 / 2$ and $S=1$ spin models. The detailed calculations are presented in the Appendix.

\section{KANAMORI INTERACTION AND TIGHT BINDING HAMILTONIAN}

The honeycomb network is made of metal (M) $d$-orbital sites with half-filled $t_{2 \mathrm{~g}}$ orbitals and octahedra cages of nonmagnetic ligand $(\mathrm{X})$ sites with fully occupied $p$ orbitals. The full Hamiltonian is composed of the on-site Kanamori interaction and the tight binding Hamiltonian between two sites.

The on-site Hamiltonian of the M sites is described by the Kanamori interaction [56] as well as crystal field splitting:

$$
\begin{aligned}
H_{e e}= & U \sum_{\alpha} n_{\alpha \uparrow} n_{\alpha \downarrow}+\frac{U^{\prime}}{2} \sum_{\substack{\alpha \neq \beta, \sigma, \sigma^{\prime}}} n_{\alpha \sigma} n_{\beta \sigma^{\prime}} \\
& -\frac{J_{H}}{2} \sum_{\substack{\alpha \neq \beta, \sigma, \sigma^{\prime}}} c_{\alpha \sigma}^{\dagger} c_{\beta \sigma^{\prime}}^{\dagger} c_{\beta \sigma} c_{\alpha \sigma^{\prime}}+J_{H} \sum_{\alpha \neq \beta} c_{\alpha \uparrow}^{\dagger} c_{\alpha \downarrow}^{\dagger} c_{\beta \downarrow} c_{\beta \uparrow} \\
& +\Delta_{c} \sum_{\substack{\alpha \in e_{g}, \sigma}} c_{\alpha \sigma}^{\dagger} c_{\alpha \sigma},
\end{aligned}
$$

where the density operator $n_{\alpha \sigma}$ is given by $c_{\alpha \sigma}^{\dagger} c_{\alpha \sigma}$ and $c_{\alpha \sigma}^{\dagger}$ is the creation operator with $\alpha$ orbital and spin $\sigma . U$ and $U^{\prime}$ are the intraorbital and interorbital Hubbard interaction respectively, and $J_{H}$ is the Hund's coupling for the spin-exchange and pair-hopping terms. $\Delta_{c}$ is a crystal field splitting on the $\mathrm{M}$ sites, originated from the surrounding octahedra, leading to the splitting of the $d$ orbitals into $t_{2 \mathrm{~g}}$ and $e_{\mathrm{g}}$ orbitals. In a $d^{3}$ system, one has half-filled $t_{2 \mathrm{~g}}$ orbitals, where the Hund's coupling $J_{H}$ selects for the $S=3 / 2$ configuration as the ground state, and the angular momentum is quenched. A table of the excited state energy spectrum is shown in Sec. 1 of the Appendix. The energies of the excited states are larger than the

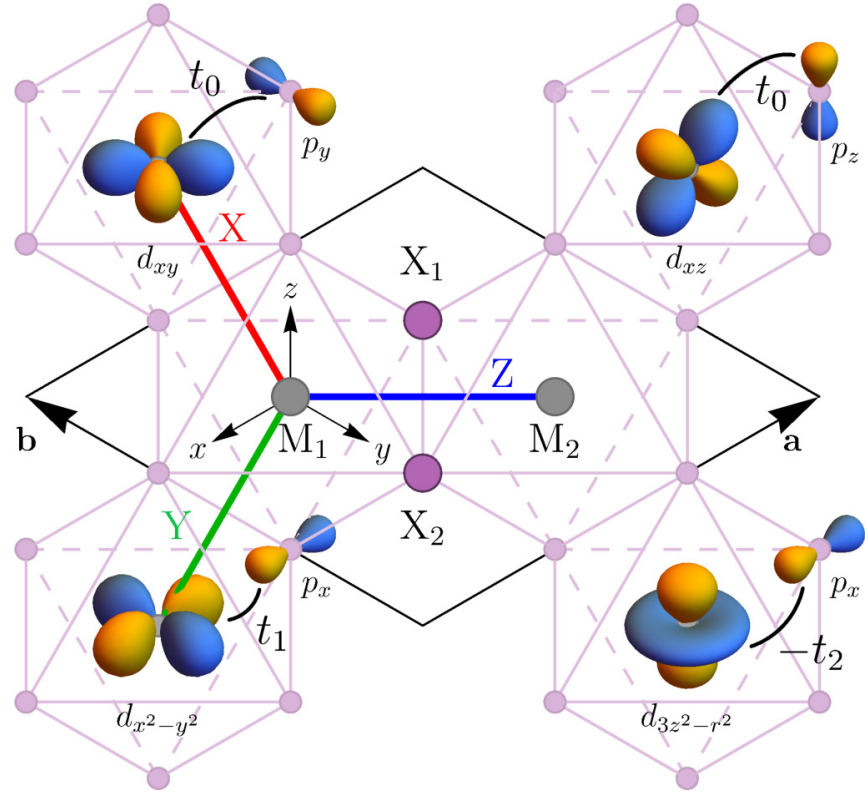

FIG. 1. Edge-shared octahedra honeycomb structure unit cell $\mathbf{a}, \mathbf{b}$, in global coordinates $x y z$. Transition metal sites $\mathrm{M}$ are in gray, and nonmagnetic ligands $\mathrm{X}$ are in purple. The NN bonds $\mathrm{X}, \mathrm{Y}$, and $\mathrm{Z}$ are related by $C_{3}$ symmetry. The sites $\mathrm{M}_{1}, \mathrm{M}_{2}, \mathrm{X}_{1}$, and $\mathrm{X}_{2}$ are involved in the second-order strong-coupling expansion on the $\mathrm{Z}$ bond. Indirect hopping integrals $t_{0}, t_{1}$, and $t_{2}$ are shown.

hopping integrals, which allows us to treat the tight binding hopping integrals as a perturbation.

In the edge-shared octahedra structure, each bond between NN M sites involves two adjacent ligands, as shown in Fig. 1. A tight binding Hamiltonian between two transition metal sites $\mathrm{M}_{1}$ and $\mathrm{M}_{2}$ including the two adjacent ligands $\mathrm{X}_{1}$ and $\mathrm{X}_{2}$ is given below:

$$
H_{T B}=\left(\begin{array}{llll}
0_{5 \times 5} & \mathbf{T}_{\mathbf{M}_{\mathbf{1}} \mathbf{M}_{\mathbf{2}}} & \mathbf{T}_{\mathbf{M}_{\mathbf{1}} \mathbf{X}_{\mathbf{1}}} & \mathbf{T}_{\mathbf{M}_{\mathbf{1}} \mathbf{X}_{\mathbf{2}}} \\
\mathbf{T}_{\mathbf{M}_{\mathbf{1}} \mathbf{M}_{\mathbf{2}}}{ }^{\dagger} & 0_{5 \times 5} & \mathbf{T}_{\mathbf{M}_{\mathbf{2}} \mathbf{X}_{\mathbf{1}}} & \mathbf{T}_{\mathbf{M}_{\mathbf{2}} \mathbf{X}_{\mathbf{2}}} \\
\mathbf{T}_{\mathbf{M}_{\mathbf{1}} \mathbf{X}_{\mathbf{1}}}^{\dagger} & \mathbf{T}_{\mathbf{M}_{\mathbf{2}} \mathbf{X}_{\mathbf{2}}}^{\dagger} & 0_{3 \times 3} & 0_{3 \times 3} \\
\mathbf{T}_{\mathbf{M}_{\mathbf{1}} \mathbf{X}_{\mathbf{2}}}^{\dagger} & \mathbf{T}_{\mathbf{M}_{\mathbf{2}} \mathbf{X}_{\mathbf{2}}}^{\dagger} & 0_{3 \times 3} & 0_{3 \times 3}
\end{array}\right),
$$

where $0_{n \times n}$ refers to the $n \times n$ null matrix. The basis is chosen as $\left(C_{\mathrm{M}_{1}, d}^{\dagger}, C_{\mathrm{M}_{2}, d}^{\dagger}, C_{\mathrm{X}_{1}, p}^{\dagger}, C_{\mathrm{X}_{2}, p}^{\dagger}\right)$, where $C_{\mathrm{M}_{i}, d}^{\dagger}=$ $\left(c_{i, x^{2}-y^{2}}^{\dagger}, c_{i, 3 z^{2}-r^{2}}^{\dagger}, c_{i, y z}^{\dagger}, c_{i, x z}^{\dagger}, c_{i, x y}^{\dagger},\right)$ are five $d$ orbitals at site $\mathrm{M}_{i}$ and $C_{\mathrm{X}_{m}, p}^{\dagger}=\left(c_{m, p_{x}}^{\dagger}, c_{m, p_{y}}^{\dagger}, c_{m, p_{z}}^{\dagger}\right)$ are three $p$ orbitals at ligand site $\mathrm{X}_{m}$. Each block of indirect hopping between $\mathrm{M}_{i}$ and $\mathrm{X}_{m}$ is denoted by $\mathbf{T}_{\mathbf{M}_{\mathbf{i}} \mathbf{X}_{\mathrm{m}}}$, and the direct hopping between $\mathrm{M}$ sites is denoted by $\mathbf{T}_{\mathbf{M}_{\mathbf{1}} \mathbf{M}_{2}}$. The details of each block matrix will be presented later.

To account for the indirect $d$-to- $p$ hoppings, we integrate out the $p$ orbitals through a perturbative procedure truncated at second order, leading to an effective $d$-to- $d$ hopping model:

$$
\mathbf{T}_{\mathbf{M}_{\mathbf{i}} \mathbf{M}_{\mathbf{j}}}^{\text {eff }}=\sum_{(a, m)} \frac{\mathbf{T}_{\mathbf{M}_{\mathbf{i}} \mathbf{X}_{\mathbf{m}}}|a\rangle\langle a| \mathbf{T}_{\mathbf{X}_{\mathbf{m}} \mathbf{M}_{\mathbf{j}}}}{\Delta E_{a}},
$$

where $(a, m)$ represent a sum over all single-hole states $a$ of all sites $\mathrm{X}_{m}$. The hole states are SOC states, thus creating two energy costs $\Delta E_{a}=\Delta-\lambda_{p} / 2$ or $\Delta+\lambda_{p}$, where $\Delta=\epsilon_{d}-\epsilon_{p}$ 
is the atomic energy difference between $\mathrm{M}$ and $\mathrm{X}$ sites and $\lambda_{p}$ is the SOC in $p$ orbitals. The SOC will introduce explicit spin dependence in the effective $d$-to- $d$ hopping. The total effective hopping between the two M sites now reads

$$
H_{T B}^{\text {eff }}=\left(\begin{array}{ll}
0_{10 \times 10} & \mathbf{T}_{\mathbf{M}_{1} \mathbf{M}_{2}}+\mathbf{T}_{\mathbf{M}_{1} \mathbf{M}_{2}}^{\text {eff }} \\
\mathbf{T}_{\mathbf{M}_{1} \mathbf{M}_{\mathbf{2}}}^{\dagger}+\mathbf{T}_{\mathbf{M}_{1} \mathbf{M}_{2}}^{\text {eff } \dagger} & 0_{10 \times 10}
\end{array}\right),
$$

where we still retain the bare direct hoppings $\mathbf{T}_{\mathbf{M}_{1} \mathbf{M}_{2}}$. Below we focus on the ideal honeycomb structure and first examine the effects of $\mathbf{T}_{\mathbf{M}_{1} \mathbf{M}_{2}}^{\text {eff }}$ before adding the direct hoppings $\mathbf{T}_{\mathbf{M}_{1} \mathbf{M}_{2}}$ and summarizing the resulting spin model.

\section{IDEAL HONEYCOMB STRUCTURE}

To understand the microscopic origin of the spin model, we start with the ideal honeycomb network surrounded by perfect edge-shared octahedra. It was shown that the symmetry of the edge-shared octahedra $\mathrm{Z}$ bond allows Heisenberg $J$, Kitaev $K$, and symmetric off-diagonal $\Gamma$ interactions $[4,29,30]$. However, since their strengths depend on various exchange processes, we perform the strong-coupling perturbation theory to determine the exchange terms.

Truncating at second order in perturbation theory, we arrive at the following Heisenberg-Kitaev $(J-K)$ spin model for the ideal honeycomb octahedra:

$$
H=\sum_{\langle i j\rangle \in \gamma} J_{0} \mathbf{S}_{i} \cdot \mathbf{S}_{j}+K_{0} S_{i}^{\gamma} S_{j}^{\gamma},
$$

where $\gamma=x, y, z$ bond and $J_{0}$ and $K_{0}$ refer to Heisenberg and Kitaev interactions for the ideal octahedra.

Below we present the details of the derivation of Heisenberg and Kitaev interactions. An explanation of the absence of the $\Gamma$ interaction within the second-order perturbation theory is also discussed. The exchange processes include the contributions from both indirect and direct hoppings. We focus on the $\mathrm{Z}$ bond of the honeycomb (Fig. 1), as the other two bonds are related by $C_{3}$ symmetry.

\section{A. Superexchange path: Indirect hopping}

We first consider indirect hoppings between the $\mathrm{M}$ and $\mathrm{X}$ sites, which are the largest hopping integrals. They enter through the effective hoppings of Eq. (4). The nonzero indirect hoppings between $\mathrm{M}_{1}$ and $\mathrm{X}_{1}$ sites $t_{0}, t_{1}$, and $t_{2}$ are shown in Fig. 1. These hoppings are incorporated in $\mathbf{T}_{\mathbf{M}_{1} \mathbf{M}_{2}}^{\text {eff }}$, which can be simplified using the Slater-Koster decomposition and symmetry-related $M-X$ bonds as shown in the Appendix. There are two contributions to both Heisenberg and Kitaev interactions, i.e., each interaction is composed of two exchange terms; one is from $t_{2 g}-t_{2 g}$ hoppings, and the other is from $e_{\mathrm{g}}-t_{2 \mathrm{~g}}$ hoppings

$$
J_{0}=J_{0}^{\mathrm{t}_{2 \mathrm{~g}}}+J_{0}^{\mathrm{e}_{\mathrm{g}}}, \quad K_{0}=K_{0}^{\mathrm{t}_{2 \mathrm{~g}}}+K_{0}^{\mathrm{e}_{\mathrm{g}}},
$$

where the superscript $t_{2 g}$ and $e_{g}$ refer to its corresponding hopping processes. Below we present each exchange path leading to $J_{0}^{\mathrm{t}_{\mathrm{g}}}, K_{0}^{\mathrm{t}_{\mathrm{g}} \mathrm{g}}, J_{0}^{\mathrm{e}_{\mathrm{g}}}$, and $K_{0}^{\mathrm{e}_{\mathrm{g}}}$.

\section{1. $t_{2 \mathrm{~g}}-t_{2 \mathrm{~g}}$ contributions}

Introducing the effective hopping integral $t_{\mathrm{eff}}=$ $\frac{t_{0}^{2}}{3}\left(\frac{2}{\Delta-\lambda_{p} / 2}+\frac{1}{\Delta+\lambda_{p}}\right)$ between $\mathrm{M}_{1}$ and $\mathrm{M}_{2}$ via $p$ orbitals, and the ratio $r=\frac{2 \lambda_{p}}{2 \Delta+\lambda_{p}}$ between SOC $\lambda_{p}$ and the atomic energy difference $\Delta$, the hopping matrix involving only $t_{2 \mathrm{~g}}$ orbitals, denoted by $t_{0}$ in Fig. 1, can be simplified in block form to

$$
\mathbf{T}_{\mathbf{M}_{1} \mathbf{M}_{2}}^{\mathrm{eff}}\left(t_{2 \mathrm{~g}} \otimes t_{2 \mathrm{~g}}\right)=t_{\mathrm{eff}}\left(\begin{array}{rrr}
0_{2 \times 2} & \sigma_{o} & i \frac{r}{2} \sigma_{x} \\
\sigma_{o} & 0_{2 \times 2} & -i \frac{r}{2} \sigma_{y} \\
-i \frac{r}{2} \sigma_{x} & i \frac{r}{2} \sigma_{y} & 0_{2 \times 2}
\end{array}\right),
$$

where $t_{2 \mathrm{~g}}=\left\{d_{y z}, d_{x z}, d_{x y}\right\}$ and $\sigma_{i}$ with $i=x, y, z$ are the Pauli matrices carrying the spin degrees of freedom and $\sigma_{o}$ is the $2 \times 2$ identity matrix. The holes in the intermediate states at the $\mathrm{X}$ site and their indirect hopping $\mathbf{T}_{\mathbf{M}_{\mathbf{i}} \mathbf{X}_{\mathbf{m}}}$ determine the type of $\sigma$ matrices in the effective hopping. For example, in the limit $\lambda_{p} \rightarrow 0$, only the $d_{y z}-d_{x z}$ term $t_{\text {eff }} \sigma_{0} \rightarrow t_{0}^{2} / \Delta$ is present which contributes to the direct hopping channel. The new terms present for nonzero $\lambda_{p}$ are the spin-flip (SF) terms between $d_{y z} / d_{x z}$ and $d_{x y}$. Such terms would normally not appear in the second-order perturbation process, as they involve a $d_{y z} / d_{x z}-p_{z}$ hopping followed by a $p_{x} / p_{y}-d_{x y}$ hopping, which will only occur if $p_{z}$ is entangled with $p_{x} / p_{y}$. The SOC among the $p_{x}, p_{y}, p_{z}$ generates such entanglement. Furthermore, when SOC is the dominant energy scale of the hole states, the wave functions are inevitably mixtures of $p$ orbitals and their spin, leading to $\sigma_{i}$ dependence proportional to the $r$ ratio of the SOC and atomic energy difference.

The superexchange process involving only $t_{2 \mathrm{~g}}$ orbitals [Eq. (7)] results in

$$
J_{0}^{\mathrm{t}_{2 g}}=\frac{8 t_{\mathrm{eff}}^{2}}{9\left(U+2 J_{H}\right)}, \quad K_{0}^{\mathrm{t}_{\mathrm{2g}}}=-\frac{4\left(r t_{\mathrm{eff}}\right)^{2}}{9\left(U+2 J_{H}\right)} .
$$

The spin-dependent hoppings have generated a $S_{i}^{z} S_{j}^{z} \mathrm{Ki}$ taev interaction. This can be rudimentarily understood by the following steps. To simplify the steps, we focus on one spin- $1 / 2$ electron hopping along the $\mathrm{Z}$ bond, through only a SF hopping. Imagining two sites starting in the $(\uparrow, \uparrow)$ state, the SF hopping can lower the energy by the following process: $(\uparrow, \uparrow)-\mathrm{SF}-(0, \downarrow \uparrow)-\mathrm{SF}-(\uparrow, \uparrow)$, at an energy cost of $-\left(r t_{\text {eff }}\right)^{2} / U$. On the other hand, if the two sites start in $(\uparrow, \downarrow)$, the SF process is forbidden from the Pauli exclusion principle. Thus the exchange path starting from $(\uparrow, \uparrow)$ and ending in $(\uparrow, \uparrow)$ lowers the energy, which generates the FM $S_{i}^{z} S_{j}^{z}$ interaction. Carrying out the details for $S=3 / 2$ leads to the expression of the spin-exchange terms shown in Eq. (8).

Among the symmetry-allowed terms, the symmetric offdiagonal term with operator $S_{i}^{x} S_{j}^{y}+S_{i}^{y} S_{j}^{x}=\frac{i}{2}\left(S_{i}^{-} S_{j}^{-}-S_{i}^{+} S_{j}^{+}\right)$ does not occur in the second-order perturbation results. This operator would connect $(\uparrow, \uparrow)$ to $(\downarrow, \downarrow)$, which is definitely possible from a SF process; however, there is a subtle cancellation. Having a single Pauli matrix in the SF term creates such cancellation among the two paths within the spin block, resulting in a null $\Gamma$ term. Thus it becomes finite only when higher-order perturbation terms are included, or when the octahedra are no longer ideal, as we will show later in Sec. IV. 


\section{2. $e_{\mathrm{g}}-t_{2 \mathrm{~g}}$ contributions}

Given that the $p$ orbital's hybridization with $e_{\mathrm{g}}$ is larger than with $t_{2 \mathrm{~g}}$, denoted by $t_{1}$ and $t_{2}$ in Fig. 1 , the $e_{\mathrm{g}}-t_{2 \mathrm{~g}}$ contribution is essential. The final form of the effective $\mathbf{T}_{\mathbf{M}_{1} \mathbf{M}_{2}}^{\text {eff }}\left(e_{\mathrm{g}} \otimes\right.$ $t_{2 \mathrm{~g}}$ ) hopping includes SF terms in the $d_{y z}, d_{x z}$ to $e_{\mathrm{g}}$ blocks, as well as spin-independent $-2 t_{\mathrm{eff}}\left(t_{2} / t_{0}\right) \sigma_{0}$ hopping between $d_{x y}$ and $d_{3 z^{2}-r^{2}}$. The effective hopping matrix involving $e_{\mathrm{g}}-t_{2 \mathrm{~g}}$ hoppings, in block matrix form, reads

$$
\mathbf{T}_{\mathbf{M}_{1} \mathbf{M}_{2}}^{\mathrm{eff}}\left(e_{\mathrm{g}} \otimes t_{2 \mathrm{~g}}\right)=t_{\mathrm{eff}}\left(\begin{array}{ccc}
i \frac{r}{2} \frac{t_{1}}{t_{0}} \sigma_{y} & i \frac{r}{2} \frac{t_{1}}{t_{0}} \sigma_{x} & 0_{2 \times 2} \\
-i \frac{r}{2} \frac{t_{2}}{t_{0}} \sigma_{y} & i \frac{r}{2} \frac{t_{2}}{t_{0}} \sigma_{x} & -2 \frac{t_{2}}{t_{0}} \sigma_{o}
\end{array}\right)
$$

where $e_{\mathrm{g}}=\left\{d_{x^{2}-y^{2}}, d_{3 z^{2}-r^{2}}\right\}$ and $t_{2 \mathrm{~g}}=\left\{d_{y z}, d_{x z}, d_{x y}\right\}$. Carrying out the strong-coupling expansion, these hoppings lead to the additional contribution to the Heisenberg and Kitaev interactions

$$
\begin{aligned}
J_{0}^{\mathrm{e}_{\mathrm{g}}} & =-\frac{16 J_{H} t_{\mathrm{eff}}^{2}}{3\left(\Delta_{c}+U^{\prime}-J_{H}\right)\left(\Delta_{c}+U^{\prime}+3 J_{H}\right)} \frac{t_{2}^{2}}{t_{0}^{2}}, \\
K_{0}^{\mathrm{e}_{\mathrm{g}}} & =\frac{2 J_{H}\left(r t_{\mathrm{eff}}\right)^{2}}{3\left(\Delta_{c}+U^{\prime}-J_{H}\right)\left(\Delta_{c}+U^{\prime}+3 J_{H}\right)} \frac{t_{1}^{2}+t_{2}^{2}}{t_{0}^{2}} .
\end{aligned}
$$

Similar to the $t_{2 \mathrm{~g}}-t_{2 \mathrm{~g}}$ case, the SF terms contribute to the Kitaev interaction, while the spin-independent hopping, $-2 t_{\text {eff }}\left(t_{2} / t_{0}\right) \sigma_{0}$, generates a FM Heisenberg term. The FM Heisenberg interaction originates from the competition of two excited states separated by Hund's coupling, i.e., $e_{\mathrm{g}}$ paths consistent with the earlier findings obtained by first-principles calculations $[45,57]$.

\section{B. Direct hopping}

The direct hopping, denoted by $t_{d 1}, t_{d 2}, t_{d 3}$ and $\tilde{t}_{d 0}$ in $\mathbf{T}_{\mathbf{M}_{1} \mathbf{M}_{2}}$ between $M_{1}$ and $M_{2}$ is given by

$$
\begin{aligned}
& \mathbf{T}_{\mathbf{M}_{1} \mathbf{M}_{2}}\left(t_{2 \mathrm{~g}} \otimes t_{2 \mathrm{~g}}\right)=\left(\begin{array}{ccc}
t_{d 1} & t_{d 2} & 0 \\
t_{d 2} & t_{d 1} & 0 \\
0 & 0 & t_{d 3}
\end{array}\right) \otimes \sigma_{o}, \\
& \mathbf{T}_{\mathbf{M}_{1} \mathbf{M}_{2}}\left(e_{\mathrm{g}} \otimes t_{2 \mathrm{~g}}\right)=\left(\begin{array}{ccc}
0 & 0 & 0 \\
0 & 0 & \tilde{t}_{d 0}
\end{array}\right) \otimes \sigma_{o} .
\end{aligned}
$$

The Slater-Koster decomposition is explained in the Appendix. Since there are no SF hopping terms, this does not generate the Kitaev interaction, but changes the Heisenberg interaction.

\section{Summary and comments}

Combining both indirect and direct hopping contributions, the two exchange interactions for the ideal octahedra environ- ment [Eq. (5)] are found to be

$$
\begin{aligned}
J_{0}= & \frac{4\left[2 t_{d 1}^{2}+2\left(t_{\mathrm{eff}}+t_{d 2}\right)^{2}+t_{d 3}^{2}\right]}{9\left(U+2 J_{H}\right)} \\
& -\frac{4 J_{H}\left[2 t_{\mathrm{eff}}\left(t_{2} / t_{0}\right)-\tilde{t}_{d 0}\right]^{2}}{3\left(\Delta_{c}+U^{\prime}-J_{H}\right)\left(\Delta_{c}+U^{\prime}+3 J_{H}\right)}, \\
K_{0}= & -\frac{4\left(r t_{\mathrm{eff}}\right)^{2}}{9\left(U+2 J_{H}\right)} \\
& +\frac{2 J_{H}\left(r t_{\mathrm{eff}}\right)^{2}}{3\left(\Delta_{c}+U^{\prime}-J_{H}\right)\left(\Delta_{c}+U^{\prime}+3 J_{H}\right)} \frac{t_{1}^{2}+t_{2}^{2}}{t_{0}^{2}} .
\end{aligned}
$$

Thus for the ideal honeycomb structure, the $J-K$ model is derived within the second-order perturbation theory. Some comments are useful, which will also motivate further investigation on the effects of trigonal distortion presented in the next section. Kitaev and Heisenberg interactions have been generated, while the $\Gamma$ interaction has not appeared at second order due to a subtle cancellation. The Kitaev interaction has been generated purely from SF hoppings and has a prefactor of $r^{2}=\left[2 \lambda_{p} /\left(2 \Delta+\lambda_{p}\right)\right]^{2}$, while the Heisenberg includes spin-independent hopping contributions. Nevertheless, both $K$ and $J$ have two contributions which come with opposite signs: one from $t_{2 g}$-only paths and the other from the paths involving $e_{\mathrm{g}}$. This leads to a smaller Kitaev compared with Heisenberg interaction, unless the contribution from $e_{\mathrm{g}}$ paths reduces the overall strength of the Heisenberg interaction. So long as the crystal field splitting is not excessively large, the naturally larger $e_{\mathrm{g}}$ hoppings will drive the system to a FM Heisenberg interaction.

The FM $J-K$ model pins the moment along the cubic axis when the quantum fluctuations are taken into account [58,59], not along the observed [111] direction in $\mathrm{CrI}_{3}$. We thus investigate whether other interaction terms may be generated. The $\Gamma$ interaction allowed by the symmetry can be finite if higher-order perturbation terms are included. However, one may ask whether there are other interactions allowed by a slight distortion of the lattice within the second-order perturbation theory without invoking higher-order terms. Indeed, TMT materials do not have ideal octahedra, but have either rhombohedral $R \overline{3}$ or monoclinic $C 2 / m$ structures, and their magnetism strongly depends on structural differences and the number of layers [60-63]. Below we study the effects of distorted octahedra, which induce additional hopping integrals which were forbidden without the distortion.

\section{EFFECTS OF DISTORTION: DISTORTED OCTAHEDRA}

$\mathrm{CrI}_{3}$ goes through a structural transition from $C 2 / m$ to $R \overline{3}$ structure at low temperature [48]. In the rhombohedral structure, there are two types of $\mathrm{X}$ ligand position deviations from the ideal octahedra structure. As shown in Fig. 2(a), a single octahedron can be viewed as two shaded triangles. One distortion is the staggered rotations of the two triangles denoted by $\delta x$ blue arrows, with displacements of $\mathrm{X}$ sites perpendicular to the $\hat{c}=[1,1,1] / \sqrt{3}$ direction. The other distortion is the compression of the distance between these two triangles along the $\hat{c}$ axis denoted by $\delta x^{\prime}$ orange arrows, with displacements along the $\hat{c}$ direction. Here, the dimensionless parameters $\delta x$ and $\delta x^{\prime}$ are in units of the distance between the NN M sites 


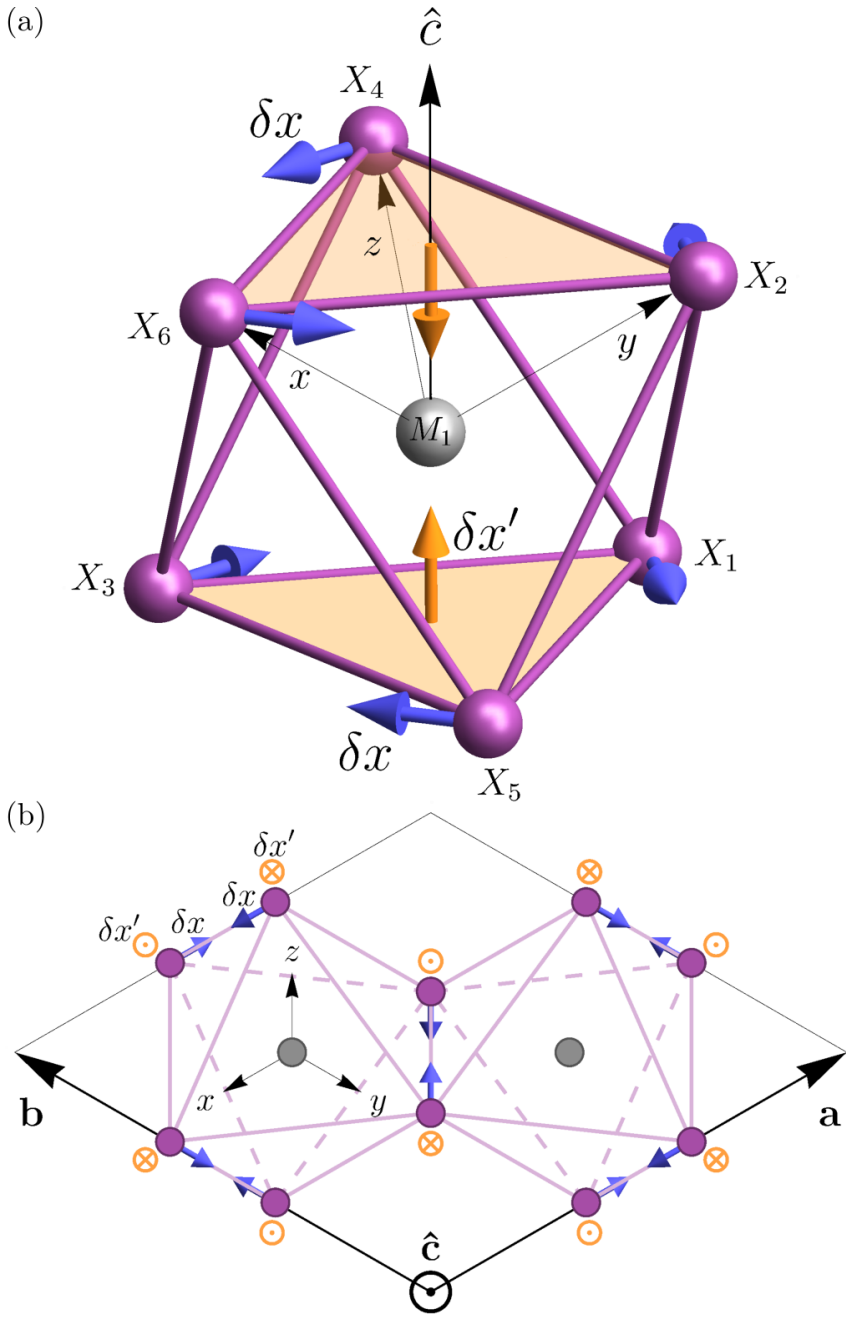

FIG. 2. (a) The distorted octahedra in $R \overline{3}$ are shown. The octahedron made of $\mathrm{X}$ ligand can be viewed as two yellow triangles normal to the $\hat{c}=[1,1,1] / \sqrt{3}$ direction. The blue arrows represent new positions of $\mathrm{X}$ due to the staggered rotations of the two yellow triangular faces. The change of position $\mathrm{X}$ due to the staggered rotations is parametrized by $\delta x$. In addition, there is a compression of the two yellow triangles, squeezed as shown by the orange arrows parallel to the $\hat{c}$ axis. The change of position due to the compression is parametrized by $\delta x^{\prime}$. (b) A top view of distortions in a unit cell is shown. The dotted circles $\odot$ indicate the new position of $\mathrm{X}$ moving out of the page along $+\hat{c}$, while the circled crosses $\otimes$ indicate a new position of $\mathrm{X}$ moving into the page toward $-\hat{c}$. The exact positions of $\mathrm{X}_{(1,2,3,4,5,6)}$ as a function of $\delta x, \delta x^{\prime}$ are found in the Appendix.

$d_{M}$. Analytic formulas of the new positions of $\mathrm{X}$ sites under staggered rotations and compression are found in Sec. 3 of the Appendix. In the $R \overline{3}$ space group there are other types of distortions; namely, the $\mathrm{M}_{1}-\mathrm{X}_{2,4,6}$ bond length can be different from the $\mathrm{M}_{1}-\mathrm{X}_{1,3,5}$ bond length. This type of distortion is generally exceedingly small compared with $\delta x$ and $\delta x^{\prime}$, and we neglect it in the following analysis. Figure 2(b) shows a top view of the honeycomb unit cell with two such distorted octahedra forming the $\mathrm{Z}$ bond, and the staggered rotation of the right octahedron is the mirror image of the left octahedron.

The distortion-induced hopping matrices have all elements nonzero in $\mathbf{T}_{\mathbf{M}_{\mathbf{i}} \mathbf{X}_{\mathbf{m}}}$ as a result of lowering the local symme-
TABLE I. Indirect and direct hoppings in units of $\mathrm{meV}$ obtained by $a b$ initio calculations. The hopping integrals are defined in Eqs. (A1) and (11).

\begin{tabular}{lcccccc}
\hline \hline$t_{0}$ & $t_{1}$ & $t_{2}$ & $t_{d 1}$ & $t_{d 2}$ & $t_{d 3}$ & $\tilde{t}_{d 0}$ \\
\hline 590.1 & -992.13 & -558.3 & 44.67 & -41.26 & -147.44 & -20.75
\end{tabular}

try of the octahedron from $O_{h}$ to $D_{3}$. We denote the new distortion-allowed hopping integrals as $\delta t_{i}$. Starting with the distortion-induced hoppings, we follow the procedure described in Sec. III; namely, we use the distortion-induced $\mathbf{T}_{\mathbf{M}_{\mathrm{i}} \mathbf{X}_{\mathbf{m}}}$ matrices to derive the effective $\mathbf{T}_{\mathbf{M}_{1} \mathbf{M}_{2}}^{\text {eff }}\left(t_{2 \mathrm{~g}} \otimes t_{2 \mathrm{~g}}\right)$ and $\mathbf{T}_{\mathbf{M}_{\mathbf{1}} \mathbf{M}_{\mathbf{2}}}^{\text {eff }}\left(e_{\mathrm{g}} \otimes t_{2 \mathrm{~g}}\right)$. Details of their form are deferred to Sec. 4 of the Appendix. Treating the distortion-induced effective hoppings as a perturbation against the on-site interactions [Eq. (1)], the minimal NN spin model is finally given by

$$
\begin{aligned}
H= & \sum_{\langle i j\rangle \in \alpha \beta(\gamma)}\left[J \mathbf{S}_{i} \cdot \mathbf{S}_{j}+K S_{i}^{\gamma} S_{j}^{\gamma}+\Gamma\left(S_{i}^{\alpha} S_{j}^{\beta}+S_{i}^{\beta} S_{j}^{\alpha}\right)\right. \\
& \left.+\Gamma^{\prime}\left(S_{i}^{\alpha} S_{j}^{\gamma}+S_{i}^{\beta} S_{j}^{\gamma}+S_{i}^{\gamma} S_{j}^{\alpha}+S_{i}^{\gamma} S_{j}^{\beta}\right)\right] \\
& +\sum_{i} A_{c}\left(\mathbf{S}_{i} \cdot \hat{\mathbf{c}}\right)^{2}
\end{aligned}
$$

where $\alpha, \beta,(\gamma)$ refers to the $\gamma$ bond taking $\alpha$ and $\beta$ spin components $[4,55]$, and $\hat{\mathbf{c}}=[1,1,1] / \sqrt{3}$. In addition to the $J-K$ terms, two symmetric off-diagonal terms $\Gamma$ and $\Gamma^{\prime}$ have been generated, as well as the single-ion term $A_{c}$ allowed by the $C_{3}$ symmetry present on every site. $\Gamma, \Gamma^{\prime}$, and $A_{c}$ are proportional to distortion-induced hoppings $\delta t$ as well as $r$; thus both distortion-induced hoppings and SOC are needed to give rise to these terms. The forms of the spin model $J-K-\Gamma-\Gamma^{\prime}-A_{c}$ to leading order in $\delta t_{i}$ as well as $e_{\mathrm{g}}$ contributions are listed in Table VI in the Appendix. Note that both Heisenberg and Kitaev interactions are renormalized by the distortion, but the Heisenberg interaction has a linear term in $\delta t$, while the Kitaev interaction does not.

\section{APPLICATION TO $\mathrm{CrI}_{3}$}

To apply the above model to understand the magnetism in $\mathrm{CrI}_{3}$, we use ab initio calculations to obtain the microscopic parameters. The calculation is performed with Vienna $a b$ initio simulation package (VASP) [64]. We use the PerdewBurke-Ernzerhof (PBE) functional [65] in our calculations. The experimental bulk structure [48] is used for the calculations. We use WANNIER90 [66] to extract the hopping integrals.

From the density functional theory results, we estimate the crystal field splitting $\Delta_{c}=1253 \mathrm{meV}$ from on-site $\mathrm{Cr} d$ orbitals as well as a crystal field splitting from the on-site I $p$ orbitals of $\Delta_{p}=528 \mathrm{meV}$. From $\mathrm{Cr} d$ and I $p$ orbitals

TABLE II. Distortion-induced indirect hoppings in units of meV obtained by $a b$ initio calculations. The hopping integrals are defined in Eq. (A5).

\begin{tabular}{ccccccccc}
\hline \hline$\delta t_{1}$ & $\delta t_{2}$ & $\delta t_{3}$ & $\delta t_{4}$ & $\delta t_{5}$ & $\delta t_{1}^{\prime}$ & $\delta t_{2}^{\prime}$ & $\delta t_{3}^{\prime}$ & $\delta t_{4}^{\prime}$ \\
\hline 2.34 & 21.76 & -22.81 & -61.74 & 65.86 & 70.97 & -43.50 & -49.41 & 29.65
\end{tabular}




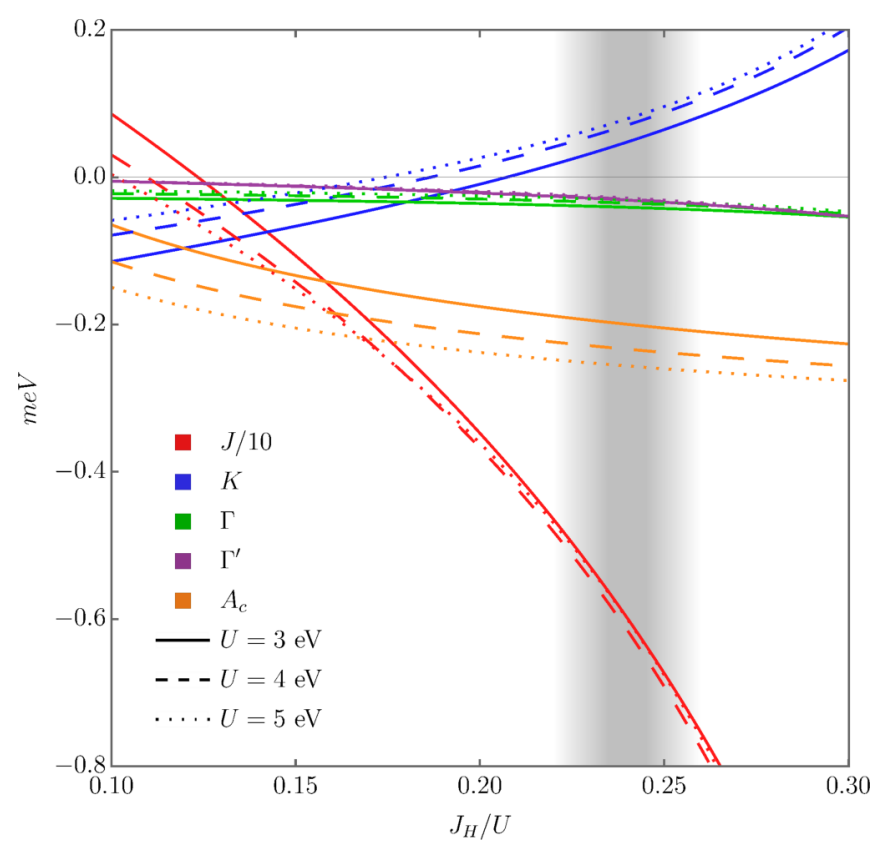

FIG. 3. Spin model parameters $J, K, \Gamma, \Gamma^{\prime}$, and $A_{c}$ estimated from $a b$ initio parameters and plotted against $J_{H} / U$. Solid, dashed, and dotted lines correspond to $U=3 \mathrm{eV}, 4 \mathrm{eV}$, and $5 \mathrm{eV}$, respectively. The shaded region corresponds to $J_{H} / U \sim 0.24$, which is relevant to $\mathrm{CrI}_{3}$ microscopics.

we extract the atomic energy difference between $\mathrm{Cr}$ and I, $\Delta=2070 \mathrm{meV}$. Finally, we find the dominant indirect $p-d$ hopping integrals $t_{0}, t_{1}$, and $t_{2}$ and the direct $d-d$ hopping parameters, listed in Table I, as well as the distortion-induced $p-d$ hoppings, listed in Table II. We verified that $t_{0}, t_{1}$, and $t_{2}$ obtained by the $a b$ initio calculation match well with the Slater-Koster expectations of Eq. (A2).

In the $a b$ initio calculations, we find a sizable crystal field splitting $\Delta_{p}$ on the ligand I sites, which we have not taken into account in the earlier analysis. It is about $528 \mathrm{meV}$, which is comparable to an estimated SOC parameter of $\lambda_{p}=630 \mathrm{meV}$. To capture its effects, we revisit the effective hopping derivation and add the crystal field splitting $\Delta_{p}$ on the $\mathrm{X}$ sites. Then the energy level in Eq. (3) splits into three levels as $\Delta E_{a}=$ $\Delta-\frac{\lambda}{2}, \Delta+\frac{1}{4}\left(2 \Delta_{p}+\lambda \pm \sqrt{\left(2 \Delta_{p}-\lambda\right)^{2}+8 \lambda^{2}}\right)$. While the holes in I sites are no longer pure total angular momentum states, the SOC is sizable enough to carry the spin entanglement through to the spin model. We found that second-order perturbation theory, including the splitting $\Delta_{p}$ in the effective hoppings, does not change the form of the spin model [Eq. (13)]. The analytic formulas would be vastly more complex in this case, so we proceed to a direct numerical evaluation of $J-K-\Gamma-\Gamma^{\prime}-A_{c}$ exchange terms using effective hoppings $\mathbf{T}_{\mathbf{M}_{1} \mathbf{M}_{2}}^{\text {eff }}$ obtained by the $a b$ initio calculations with results listed in Tables I and II.

Assuming the spherical symmetry, i.e., $U^{\prime}=U-2 J_{H}$, we are left with two unknown parameters, $U$ and $J_{H}$. We plot $J$, $K, \Gamma, \Gamma^{\prime}$, and $A_{c}$ as a function of $J_{H} / U$ for several $U$ values, with results shown in Fig. 3. The dominant term is Heisenberg except near the range $0.11 \lesssim J_{H} / U \lesssim 0.13$, where $J$ is almost zero before it changes sign. The sign of $J(K)$ is sensitive to the ratio $J_{H} / U$, and an adequately large Hund's coupling allows

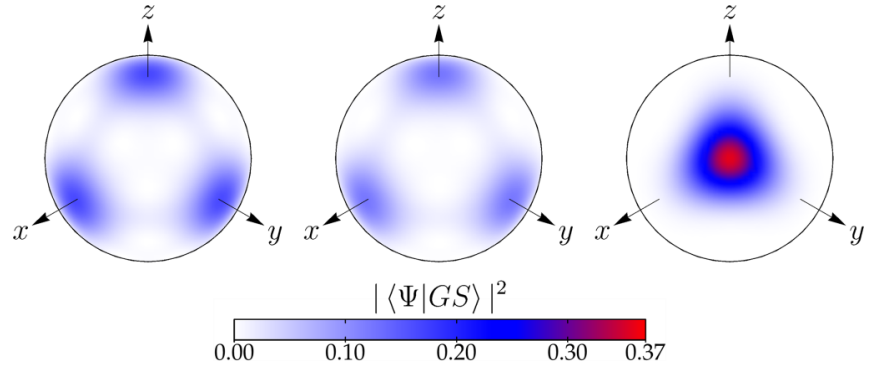

FIG. 4. Moment pinning calculation for different values of $(J, K, \Gamma)$ in the ferromagnetic phase: (a) $(-1.00,-0.20,0.00)$, (b) $(-0.20,-1.00,0.00)$, and (c) $(-0.20,-1.00,-0.02)$. The Kitaev interaction always prefers the cubic axis in (a) and (b). A small interaction such as $\Gamma$ is necessary to pin along the $\hat{c}$ direction as shown in (c).

the FM Heisenberg (AFM Kitaev) interaction to persevere. This reflects the competition of the $t_{2 \mathrm{~g}}$ vs $e_{\mathrm{g}}$ terms seen in Eq. (12), with $e_{\mathrm{g}}$ eventually winning over $t_{2 \mathrm{~g}}$ leading to the FM Heisenberg interaction. The distortion-induced $\Gamma-\Gamma^{\prime}-A_{c}$ come in with FM sign, and the single-ion anisotropy $A_{c}$ is the sizable term. Adopting the ratio of $J_{H} / U \sim 0.24$ obtained by constrained random phase approximation (cRPA) calculations [67], $\mathrm{CrI}_{3}$ sits in the shaded area in Fig. 3. $\mathrm{CrI}_{3}$ is a ferromagnet with non-negligible $K-\Gamma-\Gamma^{\prime}-A_{c}$, and we now examine the implication of these exchange terms for the observed moment.

\section{MAGNETIC ANISOTROPY}

We have shown how the ideal honeycomb structure leads to the $J-K$ model up to second order in perturbation, while distortions additionally generate $\Gamma-\Gamma^{\prime}-A_{c}$. The magnetic moment of the ferromagnetic state obtained with the FM $J-K$ model is pinned along the cubic axis such as [100] and $C_{3}$ equivalent directions via quantum fluctuations $[58,59]$. We take a closer look at the effects of $\Gamma-\Gamma^{\prime}-A_{c}$ on the moment direction. We show three examples in Fig. 4 to illustrate the moment pinning direction.

Following the method in Ref. [59], we perform exact diagonalization calculations on an eight-site honeycomb cluster, with the cluster setup shown in the Appendix. Once the ground-state wave function $|\mathrm{GS}\rangle$ is obtained, the probability distribution $P=\left|\left\langle\Psi_{\mathrm{FM}}(\theta, \phi) \mid \mathrm{GS}\right\rangle\right|^{2}$ is computed, where $\left|\Psi_{\mathrm{FM}}(\theta, \phi)\right\rangle$ is a ferromagnetic ansatz with moment direction pointing at $(\theta, \phi)$ on the sphere. Results are shown in Fig. 4 for different values of $J, K$, and $\Gamma$. Independent of the ratio of $J$ and $K$, the moment is along the cubic axis as shown in Figs. 4(a) and 4(b). On the other hand, when a small FM $\Gamma$ is introduced, the moment is along the $\hat{c}$ direction as shown in Fig. 4(c). This effect can be anticipated from the classical analysis. The classical $J-K-\Gamma$ model in the FM state with moment $\mathbf{S}_{0}=\left(S_{0}^{x}, S_{0}^{y}, S_{0}^{z}\right)$ has an energy density per unit cell $u^{c}=(3 J+K)+2 \Gamma\left(S_{0}^{x} S_{0}^{y}+S_{0}^{y} S_{0}^{z}+S_{0}^{z} S_{0}^{x}\right)$, which demands $\min \left[u^{c}\right] \Rightarrow \min \left[\operatorname{sgn}(\Gamma)\left(S_{0}^{x} S_{0}^{y}+S_{0}^{y} S_{0}^{z}+S_{0}^{z} S_{0}^{x}\right)\right]$. When $\Gamma<0$, we have $\min \left[-\left(S_{0}^{x} S_{0}^{y}+S_{0}^{y} S_{0}^{z}+S_{0}^{z} S_{0}^{x}\right)\right] \Rightarrow \mathbf{S}_{0}=\hat{c}$ leading to a moment pinned on $[1,1,1]$. We choose $\Gamma / K \ll 1$ to show that a tiny FM $\Gamma$ anisotropy results in the $\hat{c}$-axis moment shown in Fig. 4(c). 
FM $\Gamma^{\prime}$ has an effect identical to that of FM $\Gamma$ with respect to the moment direction of the ferromagnet. Clearly, FM $A_{c}$ also promotes the $\hat{c}$-axis moment direction. All three interactions individually prefer the moment on the $\hat{c}$ axis. This effect is bigger than the quantum fluctuation effect of the cubic axis pinning from the Kitaev term resulting in the observed $\hat{c}$-axis moment direction.

\section{SPIN GAP AND FINITE TRANSITION TEMPERATURE}

The preceding results suggest that it is likely that $\mathrm{CrI}_{3}$ has dominant FM Heisenberg and smaller Kitaev interaction, and the magnetic anisotropy originates from the distortion of the octahedra together with the SOC of heavy ligands. The magnetic ordering pattern changes from bulk to films [49,63,6770], suggesting a strong coupling between magnetism and crystal structures, which further implies the importance of the distorted octahedra in the presence of SOC. Our microscopic model has an interesting connection to the previous studies. The Kitaev interaction and single-ion anisotropy in addition to the Heisenberg interaction were found in Ref. [53] using density functional theory. If $\Gamma=\Gamma^{\prime}$, the $J-\Gamma-\Gamma^{\prime}$ model maps to the XXZ model [51,52] in the $a-b-c$ crystallographic coordinate

$$
\begin{aligned}
& J \mathbf{S}_{i} \cdot \mathbf{S}_{j}+\Gamma\left(S_{i}^{x} S_{j}^{y}+S_{i}^{y} S_{j}^{x}+S_{i}^{x} S_{j}^{z}+S_{i}^{z} S_{j}^{x}+S_{i}^{y} S_{j}^{z}+S_{i}^{z} S_{j}^{y}\right) \\
& \quad \Longrightarrow J_{a b} \mathbf{S}_{i} \cdot \mathbf{S}_{j}+J_{c} S_{i}^{c} S_{j}^{c}
\end{aligned}
$$

where $J_{a b}=J-\Gamma$ and $J_{c}=3 \Gamma$.

Since the exchange parameters strongly depend on $J_{H} / U$, $\Delta_{c} / U$, and tight binding parameters, it is useful to obtain experimental inputs to determine some parameters. Inelastic neutron scattering experiments and magneto-Raman spectroscopy have reported a spin gap of approximately $0.36 \mathrm{meV}$ at the Brillouin zone (BZ) center $\Gamma$ point $[71,72]$. This is also consistent with a small anisotropy found in a ferromagnetic resonance experiment [54], which is about $0.07 \mathrm{meV}$ leading to a spin gap of $0.3 \mathrm{meV}$.

Based on our spin wave analysis using the $J-K-\Gamma-\Gamma^{\prime}-A_{c}$ model including the second-NN DM interaction, the spin wave dispersion $\omega_{\mathbf{k}}$ is expressed as $\omega_{0}+\rho k^{2}$ around the $\Gamma$ point. Here, $\omega_{0}$ and $\rho$ are the spin gap and stiffness, respectively, and they are given by

$$
\begin{aligned}
\omega_{o} & =-S\left(3 \Gamma+6 \Gamma^{\prime}+2 A_{c}\right), \\
\rho & =\frac{S}{12}\left|3 J+K-\Gamma-2 \Gamma^{\prime}-\frac{\left(K+2 \Gamma-2 \Gamma^{\prime}\right)^{2}}{2\left(2 \Gamma+4 \Gamma^{\prime}+2 A_{c}+3 J+K\right)}\right| .
\end{aligned}
$$

The details of the linear spin wave theory (LSWT) are presented in Sec. 7 of the Appendix.

Kitaev and DM interactions do not generate a gap at the $\Gamma$ point within the LSWT. The classical ferromagnetic ground state under the Kitaev and DM terms has a continuous degeneracy, and as a result expanding around this ground state in the LSWT will not result in a spin gap from these terms. The spin gap is rather small, as expected because it is originated from a combination of slightly distorted octahedra and SOC, i.e., $\Gamma, \Gamma^{\prime}$, and $A_{c}$. While small, it is essential for a finite $T_{c}$ in a single-layer $\mathrm{CrI}_{3}$. In the FM ordered phase, at low temperatures the magnons are excited, and their number is given by $N_{s}(T)=\int d^{2} k \frac{1}{e^{\beta \omega_{\mathbf{k}}-1}}=\frac{\pi}{\beta \rho} \int_{\beta \omega_{0}} \frac{d x}{e^{x}-1}$. Without the spin gap $\omega_{0}, N_{s}$ diverges in two dimensions at any temperature except $T=0$, i.e., the celebrated Mermin-Wagner theorem [50]. Thus one can understand the essential role of $\omega_{0}$, which cuts the divergence and allows the FM ordering at finite temperatures, as long as $\omega_{0}(T)$ remains finite for $T \leqslant T_{c}$. While quantifying the transition temperature requires further analysis [73], the temperature dependence of $\rho(T)$ and $\omega_{0}(T)$ from inelastic neutron scattering measurements [71] indicates the crucial role of $\omega_{0}(T)$ which vanishes at $T_{c}$.

Another important parameter is the Kitaev interaction $K$, which leads to a gap at the the BZ corner $K$ point known as the Dirac gap [54], reported in the neutron scattering [71,74]. However, the second-NN DM term also generates the Dirac gap $[71,74]$. We would like to point out that $\Gamma$ and $\Gamma^{\prime}$ also play a part in the Dirac gap as shown in Eq. (A14). Estimating the Kitaev interaction by an independent experimental measurement and further analysis of the individual role of the Kitaev and DM interactions remain to be resolved in future studies.

\section{SUMMARY AND DISCUSSION}

We have shown a microscopic derivation of the NN spin model for honeycomb Mott insulators with three electrons in $t_{2 \mathrm{~g}}$ orbitals at $\mathrm{M}$ sites surrounded by octahedral cages of ligands $\mathrm{X}$ with strong SOC. Using the standard strong-coupling perturbation theory, we found that there are only Heisenberg and Kitaev interactions for the ideal honeycomb lattice among the three symmetry-allowed interactions $(J, K, \Gamma)$, because $\Gamma$ is zero up to the second-order perturbation. The exchange paths between $t_{2 \mathrm{~g}}$ and $t_{2 \mathrm{~g}}$ vs between $t_{2 \mathrm{~g}}$ and $e_{\mathrm{g}}$ via ligands generate opposite signs for both Heisenberg and Kitaev interactions. The Heisenberg interaction is of order $t_{\text {eff }}^{2} / U$, while the Kitaev interaction is smaller by a factor of $r^{2} \sim\left(\lambda_{p} / \Delta\right)^{2}$. The FM Heisenberg interaction originates from the $e_{\mathrm{g}}$ paths, with the hopping integral between $e_{\mathrm{g}}$ and $p$ orbitals being larger compared with that between $t_{2 \mathrm{~g}}$ and $p$ orbitals.

The FM Heisenberg and Kitaev interaction leads to FM ordering, but the moment direction is pinned along the cubic $x, y$, or $z$ axis, e.g., [100] (and $C_{3}$ equivalent directions) via quantum fluctuations. The $\hat{c}$-axis, [111], moment pinning found in $\mathrm{CrI}_{3}$ should thus originate from other interactions, which are also responsible for the spin gap at the $\Gamma$ point in neutron scattering measurements [71]. Including the distorted octahedra present in the rhombohedral structure, three additional spin interactions are found. Inspecting the linear order in the distortion-induced hopping paths within the secondorder perturbation theory, $\Gamma, \Gamma^{\prime}$, and single-ion anisotropy $A_{c}$ contain terms that are linear in the distortion-induced hopping integrals. The Heisenberg interaction also contains such an additional linear term, but the Kitaev interaction does not, implying that it is possible to fine-tune a system closer to the Kitaev-dominant regime via trigonal distortions.

In this paper, we have focused on the nature of the monolayer; however, some comments are in place when considering the physics of the bulk. We showed that the $t_{2 \mathrm{~g}}-t_{2 \mathrm{~g}}$ vs $e_{\mathrm{g}}-t_{2 \mathrm{~g}}$ contributions to the intralayer Heisenberg term $J$ are opposite in sign. This property should hold for interlayer Heisenberg interaction $J_{\perp}$ as well, because it is determined from su- 
TABLE III. The leading term of the exchange interactions for the ideal honeycomb structure in units of $t_{\text {eff }}^{2} / U$ for different spin $S$ including only up to the second-order perturbation terms. See the Appendix for the full expression of $J-K-\Gamma-\Gamma^{\prime}-A_{c}$ for $S=3 / 2$ including the trigonal distortion-induced hopping contributions.

\begin{tabular}{lccc}
\hline \hline Spin & $\begin{array}{c}\text { Heisenberg } \\
J_{0}\end{array}$ & $\begin{array}{c}\text { Kitaev } \\
K_{0}\end{array}$ & $\begin{array}{c}\text { Symmetric } \\
\text { off-diagonal } \Gamma_{0}\end{array}$ \\
\hline$J_{\text {eff }}=1 / 2\left(d^{5}\right)$ & $O\left(\frac{t_{d}^{2}}{t_{\text {eff }}^{2}}\right)$ & $O\left(\frac{J_{H}}{U}\right)$ & $O\left(\frac{t_{d}}{t_{\text {eff }}} \frac{J_{H}}{U}\right)$ \\
$S=1\left(d^{8}\right)$ & $O\left(r^{2}\right)$ & $O\left(r^{2}\right)$ & 0 \\
$S=3 / 2\left(d^{3}\right)$ & $O(1)$ & $O\left(r^{2}\right)$ & 0 \\
\hline \hline
\end{tabular}

perexchange processes. Thus the magnetic ordering pattern between layers depends on the details of orbital compositions. From the $a b$ initio calculation, we found that the interlayer hopping ranges from 10 to $30 \mathrm{meV}$. This leads to $J_{\perp}$ of order $0.1 \mathrm{meV}$. While it does not affect the intralayer magnetism presented here, it is important for the ordering pattern in the bulk [49,63,67-70].

Comparison with $J_{\text {eff }}=1 / 2, S=1$, and $S=3 / 2$ spin systems would be useful. The SOC is necessary to generate the bond-dependent interaction, as spin and orbitals should be entangled to get such a directional-dependent spin interaction. However, the presence of SOC is not enough to find an exotic phase such as a spin liquid, because the dominant interaction is often the Heisenberg interaction. To compare different spin cases, a summary of the ideal honeycomb exchange interactions $J_{0}, K_{0}$, and $\Gamma_{0}$ including the effective indirect hopping $\left(t_{\text {eff }}\right)$ and direct hopping $\left(t_{d}\right)$ integrals, only up to the secondorder perturbation, is shown in Table III.

Focusing on the ideal octahedra and NN model via secondorder superexchange processes, $J_{\text {eff }}=1 / 2$ is unique because the Heisenberg term is absent. On the other hand, for the $S=1$ model from $d^{2}$ in $e_{\mathrm{g}}$ orbitals, the heavy ligand SOC generates the Kitaev interaction, which has the same order of magnitude as $J$. In fact, $K=-2 J$, if only $e_{\mathrm{g}}$ paths are considered [25]. For the $S=3 / 2$ case, we found that $J$ is order 1 in units of roughly $t_{\text {eff }}^{2} / U$, while $K$ is smaller by $r^{2}$. Thus it is hard to compete with the Heisenberg interaction. We speculate that this is valid for spins equal to or higher than $3 / 2$. Unlike the $S=1 / 2$ and $S=1$ cases, the Heisenberg interaction is dominant in $S=3 / 2$, but a delicate cancellation among different contributions to the Heisenberg interaction may let the Kitaev interaction overtake it place. In particular, the Heisenberg interaction is more sensitive to the distortioninduced hopping integrals than the Kitaev term as shown in Table VI in the Appendix; manipulating octahedra may be a way to tune the system to a desired Kitaev-dominant regime.

In summary, in the ideal octahedra environment, we find that there are only two spin interactions, Heisenberg and Kitaev interactions. Kitaev interaction is generally weaker compared with the Heisenberg interaction in contrast to the lower-spin models. Indirect hoppings among $t_{2 \mathrm{~g}}$ and $t_{2 \mathrm{~g}}$ vs those among $e_{\mathrm{g}}$ and $t_{2 \mathrm{~g}}$ have opposite contributions. A detailed balance between the two indirect and direct hopping contributions highly depends on the hopping integrals, Hund's coupling strength, and crystal field splitting. $\Gamma$ interaction is absent up to the second order due to a subtle cancellation. We further show that trigonal distortion allows three additional interactions: two symmetric off-diagonal interactions $\Gamma$ and $\Gamma^{\prime}$ and single-ion anisotropy $A_{c}$ along the $\hat{c}$ axis. They are all linearly proportional to a distortion-induced hopping integral. While they are much smaller than the Heisenberg interaction, they are essential for a spin gap in the FM phase of $\mathrm{CrI}_{3}$ leading to a finite $T_{c}$. Our study offers a microscopic route to the NN spin models, $J-K-\Gamma-\Gamma^{\prime}-A_{c}$ interactions. Given that there are five exchange terms within the $\mathrm{NN}$ model, and second-NN interactions including DM may be comparable to $\Gamma, \Gamma^{\prime}$, and $A_{c}$, further theoretical and experimental studies are required to determine the microscopic parameters of $\mathrm{CrI}_{3}$ beyond the NN model.

\section{ACKNOWLEDGMENTS}

We acknowledge I. Lee, C. Hammel, N. Trivedi, P. Dai, and R. Valenti for useful discussions. H.Y.K. acknowledges the funding from the Canada Research Chair Program. This work was supported by the Natural Sciences and Engineering Research Council of Canada, the Center for Quantum Materials at the University of Toronto, and the Canadian Institute for Advanced Research. Computations were performed on the Niagara supercomputer at the SciNet HPC Consortium. SciNet is funded by the Canada Foundation for Innovation under the auspices of Compute Canada, the Government of Ontario, Ontario Research Fund - Research Excellence, and the University of Toronto.

\section{APPENDIX}

\section{Energy levels of on-site Kanamori Hamiltonian}

To obtain the NN spin interaction, we used the secondorder perturbation theory, where the dominant interaction is Eq. (1). First we note that in the lowest energy state there are three electrons at each metal site $\mathrm{M}$. The exchange processes then involve one electron hopping between $\mathrm{M}$ sites. Thus the intermediate states have two electrons in $t_{2 \mathrm{~g}}$ orbitals on one $\mathrm{M}$ site and four electrons in either $t_{2 \mathrm{~g}}$ or $e_{\mathrm{g}}$ orbitals on the other M site. On the other hand, the single-ion anisotropy would follow from a single $\mathrm{M}$ site, where the three electrons in the ground state interact with an excited three-electron state. The energy levels of all states involved in these exchange processes are given in Table IV.

\section{Ideal hoppings}

In the ideal octahedron environment the allowed indirect hoppings are shown in Fig. 5. On the $\mathrm{M}_{1}-\mathrm{X}_{1}$ bond we have the hopping matrix

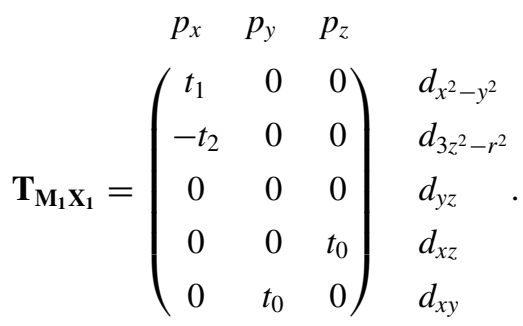

The other indirect bonds $\mathbf{T}_{\mathbf{M}_{2} \mathbf{X}_{2}}, \quad \mathbf{T}_{\mathbf{M}_{2} \mathbf{X}_{1}}, \quad \mathbf{T}_{\mathbf{M}_{1} \mathbf{X}_{2}}$ are obtained from $\mathbf{T}_{\mathbf{M}_{1} \mathbf{X}_{2}}$ by consecutively applying the symmetry 
TABLE V. Effective hoppings to leading order in distortion-induced hoppings, where $\delta t$ are defined in Eq. (A5). Distortion-induced hoppings are grouped as follows: $\delta t_{a}=\delta t_{1}-\delta t_{4}, \delta t_{b}=2 \delta t_{1}+\delta t_{4}, \delta t_{c}=\delta t_{3}+\delta t_{5}, \delta t_{d}=\delta t_{1}-\delta t_{2}, \delta t_{e}=\delta t_{1}+\delta t_{4}$, and $\delta t_{f}=\delta t_{1}+\delta t_{2}$

\begin{tabular}{lcc}
\hline \hline $\mathbf{T}_{\mathbf{M}_{1} \mathbf{M}_{2}}^{\text {eff }}\left(t_{2 \mathrm{~g}} \otimes t_{2 \mathrm{~g}}\right)=t_{\mathrm{eff}}\left(\begin{array}{ccc}T_{A} & T_{C} & T_{D} \\
T_{C}^{\dagger} & T_{A} & U T_{D} U^{\dagger} \\
T_{D}^{\dagger} & U^{\dagger} T_{D}^{\dagger} U & T_{B}\end{array}\right)$ & $\mathbf{T}_{\mathbf{M}_{1} \mathbf{M}_{2}}^{\text {eff }}\left(e_{\mathrm{g}} \otimes t_{2 \mathrm{~g}}\right)=t_{\mathrm{eff}}\left(\begin{array}{ccc}\tilde{T}_{A} & -U \tilde{T}_{A} U^{\dagger} & \tilde{T}_{C} \\
\tilde{T}_{B} & U \tilde{T}_{B} U^{\dagger} & \tilde{T}_{D}\end{array}\right)$ \\
\hline$T_{A}=\left(2 \frac{\delta t_{3}}{t_{0}}+\frac{t_{d 1}}{t_{\mathrm{eff}}}\right) \sigma_{o}$ & $U=\mathrm{e}^{i \pi \frac{1}{\sqrt{2}}\left(\frac{\sigma_{x}}{2}-\frac{\sigma_{y}}{2}\right)}=\frac{i}{\sqrt{2}}\left(\sigma_{x}-\sigma_{y}\right)$ \\
$T_{B}=\left(4 \frac{\delta t_{5}}{t_{0}}+\frac{t_{d 3}}{t_{\text {eff }}}\right) \sigma_{o}$ & $\tilde{T}_{A}=\left(\frac{\delta t_{2}^{\prime}}{t_{0}}+\frac{\delta t_{d} t_{1}}{t_{0}^{2}}\right) \sigma_{o}+i \frac{r}{2}\left(\left(\frac{\delta t_{3} t_{1}}{t_{0}^{2}}-\frac{\delta t_{1}^{\prime}}{t_{0}}\right) \sigma_{x}+\frac{t_{1}}{t_{0}} \sigma_{y}-\frac{\delta t_{e} t_{1}}{t_{0}^{2}} \sigma_{z}\right)$ \\
$T_{C}=\left(1+\frac{t_{d 2}}{t_{\mathrm{eff}}}\right) \sigma_{o}+i \frac{r}{2} \frac{\delta t_{a}}{t_{0}}\left(\sigma_{x}+\sigma_{y}\right)$ & $\tilde{T}_{B}=\left(\frac{\delta t_{4}^{\prime}}{t_{0}}-\frac{\delta t_{f} t_{2}}{t_{0}^{2}}\right) \sigma_{o}+i \frac{r}{2}\left(\left(\frac{\delta t_{3} t_{2}}{t_{0}^{2}}-\frac{\delta t_{3}^{\prime}}{t_{0}}\right) \sigma_{x}-\frac{t_{2}}{t_{0}} \sigma_{y}-\frac{\delta t_{a} t_{2}}{t_{0}^{2}} \sigma_{z}\right)$ \\
$T_{D}=\frac{\delta t_{b}}{t_{0}} \sigma_{o}+i \frac{r}{2}\left(\sigma_{x}-\frac{\delta t_{c}}{t_{0}} \sigma_{y}-\frac{\delta t_{d}}{t_{0}} \sigma_{z}\right)$ & $\tilde{T}_{C}=i \frac{r}{2}\left(\left(\frac{\delta t_{1} t_{1}}{t_{0}^{2}}-\frac{\delta t_{2}^{\prime}}{t_{0}}\right)\left(\sigma_{x}+\sigma_{y}\right)-2\left(\frac{\delta t_{5} t_{1}}{t_{0}^{2}}-\frac{\delta t_{1}^{\prime}}{t_{0}}\right) \sigma_{z}\right)$ \\
\hline
\end{tabular}

Slater-Koster formula for rotated bonds [75]. Here, we only show the leading term in each matrix element generated from the above procedure. Note that all elements become finite; the distortion-induced hopping between $t_{2 \mathrm{~g}}$ and $p$ orbitals is denoted by $\delta t_{i}$, and that between $e_{\mathrm{g}}$ and $p$ orbitals is denoted by $\delta t_{i}^{\prime}$ :

$$
\mathbf{T}_{\mathbf{M}_{1} \mathbf{X}_{\mathbf{1}}}=\left(\begin{array}{ccc}
t_{1} & \delta t_{1}^{\prime} & \delta t_{2}^{\prime} \\
-t_{2} & \delta t_{3}^{\prime} & \delta t_{4}^{\prime} \\
\delta t_{1} & \delta t_{2} & \delta t_{3} \\
\delta t_{4} & \delta t_{1} & t_{0} \\
\delta t_{5} & t_{0} & \delta t_{1}
\end{array}\right)
$$

where $\mathrm{X}_{1}$ labels the site as in Fig. 2(a). The distorted octahedron realizes the $D_{3}$ point group, which contains $C_{3}(1,1,1)$ and $C_{2}^{\prime}(-1,1,0)$ rotations. The hoppings $\mathbf{T}_{\mathbf{M}_{1} \mathbf{X}_{3}}$ and $\mathbf{T}_{\mathbf{M}_{1} \mathbf{X}_{5}}$ are recovered by applying $C_{3}(1,1,1)$ to $\mathbf{T}_{\mathbf{M}_{1} \mathbf{x}_{1}}$. Furthermore, $\mathbf{T}_{\mathbf{M}_{1} \mathbf{X}_{1}}$ relates to $\mathbf{T}_{\mathbf{M}_{1} \mathbf{X}_{2}}$ by a $C_{2}^{\prime}(-1,1,0)$. Finally, $\mathbf{T}_{\mathbf{M}_{1} \mathbf{X}_{2}}$ relates to $\mathbf{T}_{\mathbf{M}_{1} \mathbf{X}_{4}}$ and $\mathbf{T}_{\mathbf{M}_{1} \mathbf{X}_{\mathbf{6}}}$ by $C_{3}(1,1,1)$. The direct hopping integrals denoted by $\mathbf{T}_{\mathbf{M}_{1} \mathbf{M}_{2}}$ are the same as in Eq. (11). Making use of Eq. (A5), we derive effective hoppings $\mathbf{T}_{\mathbf{M}_{1} \mathbf{M}_{2}}^{\text {ef }}$ following the method in the main text [Eq. (4)]. The distortion-induced effective hoppings to leading order in $\delta t$ are listed in Table V.

\section{Single-ion anisotropy term}

Due to the distortion and SOC, the single-ion anisotropic term is also generated via the hopping to anions which can hop back to create spin-dependent on-site terms denoted by $\mathbf{T}_{\mathbf{M}_{\mathbf{1}} \mathbf{M}_{\mathbf{1}}}^{\text {eff }}=\mathbf{T}_{\mathbf{M}_{\mathbf{2}} \mathbf{M}_{2}}^{\text {eff }}$. Without the trigonal distortion, the effective hopping integrals between $t_{2 \mathrm{~g}}$ on $\mathrm{M}_{1}$ are given by

$$
\begin{aligned}
& \mathbf{T}_{\mathbf{M}_{\mathbf{1}} \mathbf{M}_{\mathbf{1}}}^{\mathrm{eff}}\left(t_{2 g} \otimes t_{2 g}\right) \\
&=t_{\mathrm{eff}}\left(\begin{array}{rrr}
4 \sigma_{o} & i r \sigma_{z} & -i r \sigma_{y} \\
-i r \sigma_{z} & 4 \sigma_{o} & i r \sigma_{x} \\
i r \sigma_{y} & -i r \sigma_{x} & 4 \sigma_{o}
\end{array}\right) .
\end{aligned}
$$

Similarly, effective hopping between $t_{2 g}$ and $e_{g}$ is found as

$$
\mathbf{T}_{\mathbf{M}_{\mathbf{1}} \mathbf{M}_{\mathbf{1}}}^{\mathrm{eff}}\left(e_{g} \otimes t_{2 g}\right)=t_{\mathrm{eff}}\left(\begin{array}{ccc}
i r \frac{t_{1}}{t_{0}} \sigma_{x} & i r \frac{t_{1}}{t_{0}} \sigma_{y} & -i 2 r \frac{t_{1}}{t_{0}} \sigma_{z} \\
i r \frac{t_{2}+t_{3}}{t_{0}} \sigma_{x} & -i r \frac{t_{2}+t_{3}}{t_{0}} \sigma_{y} & 0_{2 \times 2}
\end{array}\right) .
$$

They lead to an $\mathbf{S}_{i} \cdot \mathbf{S}_{i}$ term, which is just equal to $S(S+1)$, an irrelevant constant to the spin model. Thus there is no single-ion anisotropy without the trigonal distortion. With the trigonal distortion, the single-ion anisotropy along the $\hat{c}$ axis is generated:

$$
\begin{aligned}
A_{c}= & -\frac{4\left(r t_{\mathrm{eff}}\right)^{2}}{\left(U+3 J_{H}-U^{\prime}\right)}\left(\frac{\delta t_{\alpha}}{t_{0}}\right) \\
& -\frac{4 J_{H}\left(r t_{\mathrm{eff}}\right)^{2}}{\Delta_{c}\left(\Delta_{c}+3 J_{H}\right)}\left[\frac{t_{1}}{t_{0}}\left(\frac{\delta t_{\beta} t_{1}}{t_{0}^{2}}+\frac{\delta t_{\alpha}^{\prime}}{t_{0}}\right)\right. \\
& \left.-\frac{t_{2}+t_{3}}{t_{0}}\left(\frac{\delta t_{\beta} t_{2}+2 \delta t_{1} t_{3}}{t_{0}^{2}}+\frac{\delta t_{\beta}^{\prime}}{t_{0}}\right)\right],
\end{aligned}
$$

where $\delta t_{\alpha}=-2 \delta t_{1}+\delta t_{2}+\delta t_{3}+\delta t_{4}+\delta t_{5}, \delta t_{\beta}=\delta t_{2}+\delta t_{3}$, $\delta t_{\alpha}^{\prime}=-\delta t_{1}^{\prime}+\frac{\sqrt{3} \delta t_{4}^{\prime}-\delta t_{2}^{\prime}}{2}$, and $\delta t_{\beta}^{\prime}=-\delta t_{3}^{\prime}+\frac{\sqrt{3} \delta t_{2}^{\prime}+\delta t_{4}^{\prime}}{2}$.

\section{Spin interaction with trigonal distortion}

Putting them all together, we have Heisenberg, Kitaev, $\Gamma$, $\Gamma^{\prime}$, and single-ion anisotropy $A_{c}$ terms. Their forms to leading order in distortion-induced hoppings $\delta t$ are summarized in Table VI. Note that the Kitaev interaction does not have any linear term of distortion and $\Gamma, \Gamma^{\prime}$, and single-ion anisotropy $A_{c}$ along the $\hat{c}$ axis are finite due to both SOC and distortion. 
TABLE VI. Spin model terms, under distortions, to leading order in $\delta t$. Distortion-induced $\delta t$ is defined in Eq. (A5), and subscripts of $\delta t$ are defined in the caption of Table $\mathrm{V}$ and in the text following Eq. (A8).

\begin{tabular}{|c|c|}
\hline$t_{2 \mathrm{~g}} \otimes t_{2 \mathrm{~g}}$ & $e_{\mathrm{g}} \otimes t_{2 \mathrm{~g}}$ \\
\hline$J=\frac{4\left[2 t_{d 1}^{2}+2\left(t_{\mathrm{eff}}+t_{d 2}\right)^{2}+t_{d 3}^{2}\right]}{9\left(U+2 J_{H}\right)}+\frac{32 t_{\mathrm{eff}}}{9\left(U+2 J_{H}\right)}\left(t_{d 1} \frac{\delta t_{3}}{t_{0}}+t_{d 3} \frac{\delta t_{5}}{t_{0}}\right)$ & $-\frac{4 J_{H}\left[2 t_{\mathrm{eff}}\left(t_{2} / t_{0}\right)-\tilde{t}_{d 0}\right]^{2}}{3\left(\Delta_{c}+U^{\prime}-J_{H}\right)\left(\Delta_{c}+U^{\prime}+3 J_{H}\right)}$ \\
\hline$K=-\frac{4\left(r t_{\mathrm{eff}}\right)^{2}}{9\left(U+2 J_{H}\right)}$ & $\frac{2 J_{H}\left(r t_{\mathrm{eff}}\right)^{2}}{3\left(\Delta_{c}+U^{\prime}-J_{H}\right)\left(\Delta_{c}+U^{\prime}+3 J_{H}\right)} \frac{t_{1}^{2}+t_{2}^{2}}{t_{0}^{2}}$ \\
\hline$\Gamma=-\frac{8\left(r t_{\mathrm{eff}}\right)^{2}}{9\left(U+2 J_{H}\right)}\left(\frac{\delta t_{c}}{t_{0}}\right)$ & $-\frac{4 J_{H}\left(r t_{\text {eff }}\right)^{2}}{3\left(\Delta_{c}+U^{\prime}-J_{H}\right)\left(\Delta_{c}+U^{\prime}+3 J_{H}\right)}\left(\frac{t_{1}\left(t_{1} \delta t_{3}-t_{0} \delta t_{1}^{\prime}\right)}{t_{0}^{3}}-\frac{t_{2}\left(t_{2} \delta t_{3}-t_{0} \delta t_{3}^{\prime}\right)}{t_{0}^{3}}\right)$ \\
\hline$\Gamma^{\prime}=-\frac{4\left(r t_{\mathrm{eff}}\right)^{2}}{9\left(U+2 J_{H}\right)}\left(\frac{\delta t_{d}}{t_{0}}\right)$ & $-\frac{2 J_{H}\left(r t_{\mathrm{eff}}\right)^{2}}{3\left(\Delta_{c}+U^{\prime}-J_{H}\right)\left(\Delta_{c}+U^{\prime}+3 J_{H}\right)}\left(-\frac{t_{1}^{2} \delta t_{e}}{t_{0}^{3}}+\frac{t_{2}^{2} \delta t_{a}}{t_{0}^{3}}\right)$ \\
\hline$A_{c}=-\frac{4\left(r t_{\mathrm{eff}}\right)^{2}}{\left(U+3 J_{H}-U^{\prime}\right)}\left(\frac{\delta t_{\alpha}}{t_{0}}\right)$ & $-\frac{4 J_{H}\left(r t_{\mathrm{eff}}\right)^{2}}{\Delta_{c}\left(\Delta_{c}+3 J_{H}\right)}\left(\frac{t_{1}}{t_{0}}\left(\frac{\delta t_{\beta} t_{1}}{t_{0}^{2}}+\frac{\delta t_{\alpha}^{\prime}}{t_{0}}\right)-\frac{t_{2}+t_{3}}{t_{0}}\left(\frac{\delta t_{\beta} t_{2}+2 \delta t_{1} t_{3}}{t_{0}^{2}}+\frac{\delta t_{\beta}^{\prime}}{t_{0}}\right)\right)$ \\
\hline
\end{tabular}

\section{Spin wave theory}

We consider $J-K-\Gamma-\Gamma^{\prime}-A_{c}$ as well as the second-NN DM term $\left(\mathbf{D}_{\mathbf{c}}\right)$ :

$$
\begin{aligned}
H= & \sum_{\langle i, j\rangle \in \alpha \beta(\gamma)}\left[J \mathbf{S}_{i} \cdot \mathbf{S}_{j}+K S_{i}^{\gamma} S_{j}^{\gamma}+\Gamma\left(S_{i}^{\alpha} S_{j}^{\beta}+S_{i}^{\beta} S_{j}^{\alpha}\right)\right. \\
& \left.+\Gamma^{\prime}\left(S_{i}^{\alpha} S_{j}^{\gamma}+S_{i}^{\beta} S_{j}^{\gamma}+S_{i}^{\gamma} S_{j}^{\alpha}+S_{i}^{\gamma} S_{j}^{\beta}\right)\right] \\
& +\sum_{\langle\langle i, j\rangle\rangle} \mathbf{D}_{\mathbf{c}} \cdot\left(\mathbf{S}_{i} \times \mathbf{S}_{j}\right)+\sum_{i} A_{c}\left(\mathbf{S}_{i} \cdot \hat{\mathbf{c}}\right)^{2}
\end{aligned}
$$

where $\mathbf{D}_{\mathbf{c}}=D_{c} \operatorname{sgn}(i j) \hat{c}$ and $\operatorname{sgn}(i j)=+1$ when $i$ to $j$ points along the orange arrows in Fig. 6 . The standard HolsteinPrimakoff transformation [76] expanded to linear order in $S$

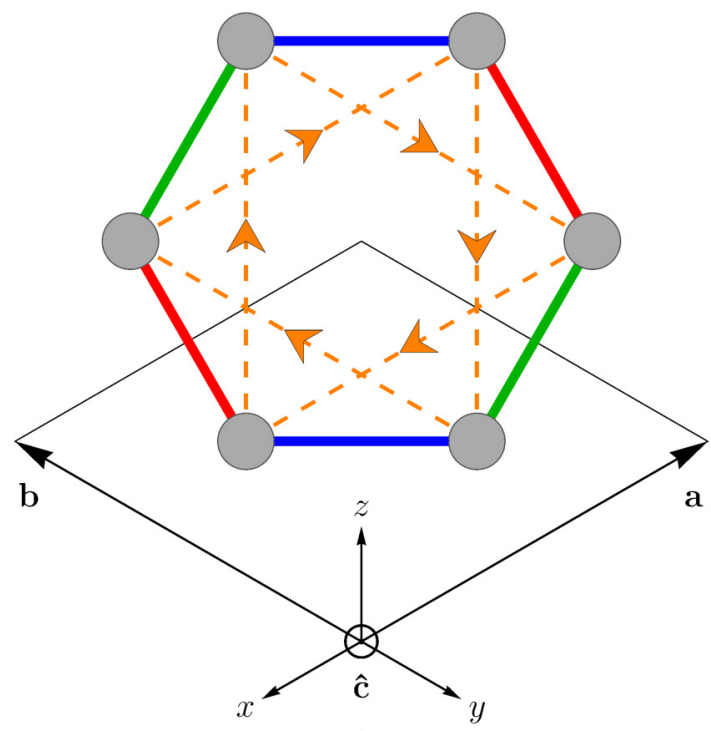

FIG. 6. X, Y, and $\mathrm{Z}$ bonds of $J-K-\Gamma-\Gamma^{\prime}-A_{c}$ shown in red, green, and blue, respectively. Second-nearest neighbors are shown in orange. The orange arrows indicate when $\operatorname{sgn}(i j)=+1$ in the DM term. reads

$$
\begin{aligned}
& S^{+}=\sqrt{2 S}\left(1-\frac{a^{\dagger} a}{2 S}\right)^{\frac{1}{2}} a \simeq \sqrt{2 S} a, \\
& S^{-}=\sqrt{2 S} a^{\dagger}\left(1-\frac{a^{\dagger} a}{2 S}\right)^{\frac{1}{2}} \simeq \sqrt{2 S} a^{\dagger}, \\
& S^{z}=S-a^{\dagger} a .
\end{aligned}
$$

Using the above and Fourier-transforming Eq. (A9) lead to

$$
\begin{aligned}
H & =E_{C L}+\sum_{\mathbf{k} \in \mathrm{BZ}} \mathrm{x}_{\mathbf{k}}^{\dagger} \mathbf{h}_{\mathbf{k}} \mathrm{x}_{\mathbf{k}}, \\
\mathbf{x}_{\mathbf{k}}^{\dagger} & =\left(a_{\mathbf{k}}^{\dagger}, b_{\mathbf{k}}^{\dagger}, a_{-\mathbf{k}}, b_{-\mathbf{k}}\right), \\
\mathbf{h}_{\mathbf{k}} & =\left(\begin{array}{cccc}
h_{o-}(\mathbf{k}) & h_{1}(\mathbf{k}) & 0 & h_{2}(\mathbf{k}) \\
h_{1}(\mathbf{k})^{*} & h_{o+}(\mathbf{k}) & h_{2}(-\mathbf{k}) & 0 \\
0 & h_{2}(-\mathbf{k})^{*} & h_{o+}(\mathbf{k}) & h_{1}(\mathbf{k}) \\
h_{2}(\mathbf{k})^{*} & 0 & h_{1}(\mathbf{k})^{*} & h_{o-}(\mathbf{k})
\end{array}\right),
\end{aligned}
$$

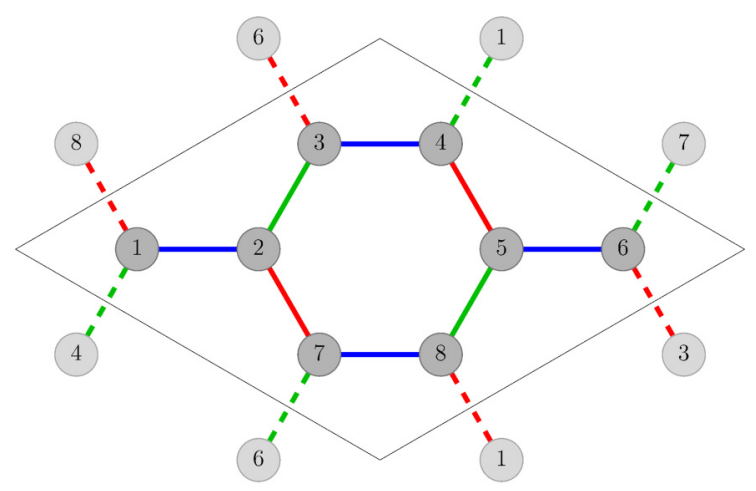

FIG. 7. X, Y, and Z bonds shown in red, green, and blue, respectively. Dashed bonds represent the periodic boundary conditions. 
where the two species of bosons $a_{\mathbf{k}}$ and $b_{\mathbf{k}}$ correspond to the two sublattices of the unit cell. The $h(\mathbf{k})$ terms are

$$
\begin{aligned}
h_{o \pm}(\mathbf{k})= & h_{o} \pm h_{\mathrm{DM}}(\mathbf{k}), \\
h_{o}= & -S\left(2 A_{c}+2 \Gamma+4 \Gamma^{\prime}+3 J+K\right), \\
h_{\mathrm{DM}}(\mathbf{k})= & 2 S D_{c}\{\sin (\mathbf{a} \cdot \mathbf{k})+\sin (\mathbf{b} \cdot \mathbf{k})-\sin [(\mathbf{a}+\mathbf{b}) \cdot \mathbf{k}]\}, \\
h_{1}(\mathbf{k})= & -\frac{S\left(\Gamma+2 \Gamma^{\prime}-3 J-K\right)}{3}\left(1+\mathrm{e}^{-i \mathbf{a} \cdot \mathbf{k}}+\mathrm{e}^{i \mathbf{b} \cdot \mathbf{k}}\right), \\
h_{2}(\mathbf{k})= & \frac{S\left(2 \Gamma-2 \Gamma^{\prime}+K\right)}{6}\left[(1-i \sqrt{3}) \mathrm{e}^{-i \mathbf{a} \cdot \mathbf{k}}\right. \\
& \left.+(1+i \sqrt{3}) \mathrm{e}^{i \mathbf{b} \cdot \mathbf{k}}-2\right],
\end{aligned}
$$

where $\mathbf{a}$ and $\mathbf{b}$ are the lattice vectors in Fig. 6. Following standard methods of diagonalizing Bogoliubov-de Gennes (BdG) Hamiltonians [77], we find the lowest eigenvalue around the $\Gamma$ point in the BZ, and upon expanding to orders of $k$, we get the spin gap $\omega_{o}$ and spin stiffness $\rho$ :

$$
\begin{aligned}
\omega_{\mathbf{k}} & =\omega_{o}+\rho k^{2}, \\
\omega_{o} & =S\left|3 \Gamma+6 \Gamma^{\prime}+2 A_{c}\right|, \\
\rho & =\frac{S}{12}\left|3 J+K-\Gamma-2 \Gamma^{\prime}-\frac{\left(K+2 \Gamma-2 \Gamma^{\prime}\right)^{2}}{2\left(2 \Gamma+4 \Gamma^{\prime}+2 A_{c}+3 J+K\right)}\right| .
\end{aligned}
$$

At the $\mathrm{K}$ point in the BZ the Dirac gap is $\omega_{+}^{K}-\omega_{-}^{K}$, where

$$
\begin{aligned}
\omega_{+}^{K}= & S\left\{\left(6 \Gamma^{\prime}+2 A_{c}+3 J\right)\left(4 \Gamma+2 \Gamma^{\prime}+2 A_{c}+3 J+2 K\right)\right. \\
& \left.+6 \sqrt{3} D_{c}\left(2 \Gamma+4 \Gamma^{\prime}+2 A_{c}+3 J+K\right)+27 D_{c}^{2}\right\}^{1 / 2}, \\
\omega_{-}^{K}= & S\left|2 \Gamma+4 \Gamma^{\prime}+2 A_{c}+3 J+K-3 \sqrt{3} D_{c}\right| .
\end{aligned}
$$

\section{Exact diagonalization calculations}

The moment pinning calculations are carried out by finding the ground state from exact diagonalization on an eight-site $(2 \times 2)$ honeycomb cluster with periodic conditions, shown in Fig. 7.
[1] M. A. McGuire, Crystal and magnetic structures in layered, transition metal dihalides and trihalides, Crystals 7, 121 (2017).

[2] G. Khaliullin, Orbital order and fluctuations in Mott insulators, Prog. Theor. Phys. Suppl. 160, 155 (2005).

[3] G. Jackeli and G. Khaliullin, Mott Insulators in the Strong SpinOrbit Coupling Limit: From Heisenberg to a Quantum Compass and Kitaev Models, Phys. Rev. Lett. 102, 017205 (2009).

[4] J. G. Rau, E. K.-H. Lee, and H.-Y. Kee, Generic Spin Model for the Honeycomb Iridates beyond the Kitaev Limit, Phys. Rev. Lett. 112, 077204 (2014).

[5] K. W. Plumb, J. P. Clancy, L. J. Sandilands, V. V. Shankar, Y. F. $\mathrm{Hu}, \mathrm{K}$. S. Burch, H.-Y. Kee, and Y.-J. Kim, $\alpha-\mathrm{RuCl}_{3}$ : A spinorbit assisted Mott insulator on a honeycomb lattice, Phys. Rev. B 90, 041112(R) (2014).

[6] H.-S. Kim, V. Shankar V., A. Catuneanu, and H.-Y. Kee, Kitaev magnetism in honeycomb $\mathrm{RuCl}_{3}$ with intermediate spin-orbit coupling, Phys. Rev. B 91, 241110(R) (2015).

[7] A. Kitaev, Anyons in an exactly solved model and beyond, Ann. Phys. (Amsterdam) 321, 2 (2006).

[8] L. J. Sandilands, Y. Tian, K. W. Plumb, Y.-J. Kim, and K. S. Burch, Scattering Continuum and Possible Fractionalized Excitations in $\alpha-\mathrm{RuCl}_{3}$, Phys. Rev. Lett. 114, 147201 (2015).

[9] J. A. Sears, M. Songvilay, K. W. Plumb, J. P. Clancy, Y. Qiu, Y. Zhao, D. Parshall, and Y.-J. Kim, Magnetic order in $\alpha-\mathrm{RuCl}_{3}$ : A honeycomb-lattice quantum magnet with strong spin-orbit coupling, Phys. Rev. B 91, 144420 (2015).

[10] R. D. Johnson, S. C. Williams, A. A. Haghighirad, J. Singleton, V. Zapf, P. Manuel, I. I. Mazin, Y. Li, H. O. Jeschke, R. Valentí, and R. Coldea, Monoclinic crystal structure of $\alpha-\mathrm{RuCl}_{3}$ and the zigzag antiferromagnetic ground state, Phys. Rev. B 92, 235119 (2015)

[11] A. Banerjee, C. A. Bridges, J.-Q. Yan, A. A. Aczel, L. Li, M. B. Stone, G. E. Granroth, M. D. Lumsden, Y. Yiu, J. Knolle, S. Bhattacharjee, D. L. Kovrizhin, R. Moessner, D. A. Tennant,
D. G. Mandrus, and S. E. Nagler, Proximate Kitaev quantum spin liquid behaviour in a honeycomb magnet, Nat. Mater. 15, 733 (2016).

[12] H. B. Cao, A. Banerjee, J.-Q. Yan, C. A. Bridges, M. D. Lumsden, D. G. Mandrus, D. A. Tennant, B. C. Chakoumakos, and S. E. Nagler, Low-temperature crystal and magnetic structure of $\alpha-\mathrm{RuCl}_{3}$, Phys. Rev. B 93, 134423 (2016).

[13] H.-S. Kim and H.-Y. Kee, Crystal structure and magnetism in $\alpha-\mathrm{RuCl}_{3}$ : An ab initio study, Phys. Rev. B 93, 155143 (2016).

[14] L. Janssen, E. C. Andrade, and M. Vojta, Magnetization processes of zigzag states on the honeycomb lattice: Identifying spin models for $\alpha-\mathrm{RuCl}_{3}$ and $\mathrm{Na}_{2} \mathrm{IrO}_{3}$, Phys. Rev. B 96, 064430 (2017).

[15] R. Yadav, N. A. Bogdanov, V. M. Katukuri, S. Nishimoto, J. van den Brink, and L. Hozoi, Kitaev exchange and field-induced quantum spin-liquid states in honeycomb $\alpha-\mathrm{RuCl}_{3}$, Sci. Rep. 6 , 37925 (2016).

[16] S.-H. Baek, S.-H. Do, K.-Y. Choi, Y. S. Kwon, A. U. B. Wolter, S. Nishimoto, J. van den Brink, and B. Büchner, Evidence for a Field-Induced Quantum Spin Liquid in $\alpha-\mathrm{RuCl}_{3}$, Phys. Rev. Lett. 119, 037201 (2017).

[17] A. U. B. Wolter, L. T. Corredor, L. Janssen, K. Nenkov, S Schönecker, S.-H. Do, K.-Y. Choi, R. Albrecht, J. Hunger, T. Doert, M. Vojta, and B. Büchner, Field-induced quantum criticality in the Kitaev system $\alpha-\mathrm{RuCl}_{3}$, Phys. Rev. B 96, 041405(R) (2017).

[18] J. Zheng, K. Ran, T. Li, J. Wang, P. Wang, B. Liu, Z.-X. Liu, B Normand, J. Wen, and W. Yu, Gapless Spin Excitations in the Field-Induced Quantum Spin Liquid Phase of $\alpha-\mathrm{RuCl}_{3}$, Phys. Rev. Lett. 119, 227208 (2017).

[19] N. Janša, A. Zorko, M. Gomilšek, M. Pregelj, K. W. Krämer, D. Biner, A. Biffin, C. Rüegg, and M. Klanjšek, Observation 
of two types of fractional excitation in the Kitaev honeycomb magnet, Nat. Phys. 14, 786 (2018).

[20] Y. Kasahara, T. Ohnishi, Y. Mizukami, O. Tanaka, S. Ma, K. Sugii, N. Kurita, H. Tanaka, J. Nasu, Y. Motome, T. Shibauchi, and Y. Matsuda, Majorana quantization and half-integer thermal quantum Hall effect in a Kitaev spin liquid, Nature (London) 559, 227 (2018).

[21] M. Yamashita, J. Gouchi, Y. Uwatoko, N. Kurita, and H. Tanaka, Sample dependence of half-integer quantized thermal Hall effect in the Kitaev spin-liquid candidate $\alpha-\mathrm{RuCl}_{3}$, Phys. Rev. B 102, 220404(R) (2020).

[22] G. Baskaran, D. Sen, and R. Shankar, Spin-S Kitaev model: Classical ground states, order from disorder, and exact correlation functions, Phys. Rev. B 78, 115116 (2008).

[23] J. Oitmaa, A. Koga, and R. R. P. Singh, Incipient and welldeveloped entropy plateaus in spin- $S$ Kitaev models, Phys. Rev. B 98, 214404 (2018).

[24] A. Koga, H. Tomishige, and J. Nasu, Ground-state and thermodynamic properties of an $S=1$ Kitaev model, J. Phys. Soc. Jpn. 87, 063703 (2018).

[25] P. P. Stavropoulos, D. Pereira, and H.-Y. Kee, Microscopic Mechanism for a Higher-Spin Kitaev Model, Phys. Rev. Lett. 123, 037203 (2019).

[26] C. Hickey, C. Berke, P. P. Stavropoulos, H.-Y. Kee, and S. Trebst, Field-driven gapless spin liquid in the spin1 Kitaev honeycomb model, Phys. Rev. Res. 2, 023361 (2020).

[27] Z. Zhu, Z.-Y. Weng, and D. N. Sheng, Magnetic field induced spin liquids in $S=1$ Kitaev honeycomb model, Phys. Rev. Res. 2, 022047(R) (2020).

[28] I. Khait, P. P. Stavropoulos, H.-Y. Kee, and Y. B. Kim, Characterizing spin-one Kitaev quantum spin liquids, Phys. Rev. Research 3, 013160 (2021).

[29] Y. Yamaji, Y. Nomura, M. Kurita, R. Arita, and M. Imada, FirstPrinciples Study of the Honeycomb-Lattice Iridates $\mathrm{Na}_{2} \mathrm{IrO}_{3}$ in the Presence of Strong Spin-Orbit Interaction and Electron Correlations, Phys. Rev. Lett. 113, 107201 (2014).

[30] V. M. Katukuri, S. Nishimoto, V. Yushankhai, A. Stoyanova, H. Kandpal, S. Choi, R. Coldea, I. Rousochatzakis, L. Hozoi, and J. van den Brink, Kitaev interactions between $j=1 / 2$ moments in honeycomb $\mathrm{Na}_{2} \mathrm{IrO}_{3}$ are large and ferromagnetic: Insights from $a b$ initio quantum chemistry calculations, New J. Phys. 16, 013056 (2014).

[31] W.-B. Zhang, Q. Qu, P. Zhu, and C.-H. Lam, Robust intrinsic ferromagnetism and half semiconductivity in stable two-dimensional single-layer chromium trihalides, J. Mater. Chem. C 3, 12457 (2015).

[32] D. Torelli and T. Olsen, Calculating critical temperatures for ferromagnetic order in two-dimensional materials, 2D Mater. 6, 015028 (2018).

[33] S. S. Pershoguba, S. Banerjee, J. C. Lashley, J. Park, H. Ågren, G. Aeppli, and A. V. Balatsky, Dirac Magnons in Honeycomb Ferromagnets, Phys. Rev. X 8, 011010 (2018).

[34] F. Zheng, J. Zhao, Z. Liu, M. Li, M. Zhou, S. Zhang, and P. Zhang, Tunable spin states in the two-dimensional magnet $\mathrm{CrI}_{3}$, Nanoscale 10, 14298 (2018).

[35] L. Webster and J.-A. Yan, Strain-tunable magnetic anisotropy in monolayer $\mathrm{CrCl}_{3}, \mathrm{CrBr}_{3}$, and $\mathrm{CrI}_{3}$, Phys. Rev. B 98, 144411 (2018).
[36] J. Liu, M. Shi, P. Mo, and J. Lu, Electrical-field-induced magnetic Skyrmion ground state in a two-dimensional chromium tri-iodide ferromagnetic monolayer, AIP Adv. 8, 055316 (2018).

[37] M. Wu, Z. Li, T. Cao, and S. G. Louie, Physical origin of giant excitonic and magneto-optical responses in two-dimensional ferromagnetic insulators, Nat. Commun. 10, 2371 (2019).

[38] T. Olsen, Theory and simulations of critical temperatures in $\mathrm{CrI}_{3}$ and other 2D materials: Easy-axis magnetic order and easy-plane Kosterlitz-Thouless transitions, MRS Commun. 9, 1142 (2019).

[39] O. Besbes, S. Nikolaev, N. Meskini, and I. Solovyev, Microscopic origin of ferromagnetism in the trihalides $\mathrm{CrCl}_{3}$ and $\mathrm{CrI}_{3}$, Phys. Rev. B 99, 104432 (2019).

[40] V. K. Gudelli and G.-Y. Guo, Magnetism and magneto-optical effects in bulk and few-layer $\mathrm{CrI}_{3}$ : A theoretical GGA $+U$ study, New J. Phys. 21, 053012 (2019).

[41] C. Xu, J. Feng, M. Kawamura, Y. Yamaji, Y. Nahas, S. Prokhorenko, Y. Qi, H. Xiang, and L. Bellaiche, Possible Kitaev Quantum Spin Liquid State in 2D Materials with $S=3 / 2$, Phys. Rev. Lett. 124, 087205 (2020).

[42] M. Pizzochero and O. V. Yazyev, Inducing magnetic phase transitions in monolayer $\mathrm{CrI}_{3}$ via lattice deformations, J. Phys. Chem. A 124, 7585 (2020).

[43] M. Pizzochero, R. Yadav, and O. V. Yazyev, Magnetic exchange interactions in monolayer $\mathrm{CrI}_{3}$ from many-body wavefunction calculations, 2D Mater. 7, 035005 (2020).

[44] E. Aguilera, R. Jaeschke-Ubiergo, N. Vidal-Silva, L. E. F. Foa Torres, and A. S. Nunez, Topological magnonics in the twodimensional van der Waals magnet $\mathrm{CrI}_{3}$, Phys. Rev. B 102, 024409 (2020).

[45] D. Soriano, M. I. Katsnelson, and J. Fernández-Rossier, Magnetic two-dimensional chromium trihalides: A theoretical perspective, Nano Lett. 20, 6225 (2020).

[46] L. L. Handy and N. W. Gregory, Structural properties of chromium(III) iodide and some chromium(III) mixed halides, J. Am. Chem. Soc. 74, 891 (1952).

[47] J. F. Dillon and C. E. Olson, Magnetization, resonance, and optical properties of the ferromagnet $\mathrm{CrI}_{3}$, J. Appl. Phys. (Melville, NY) 36, 1259 (1965).

[48] M. A. McGuire, H. Dixit, V. R. Cooper, and B. C. Sales, Coupling of crystal structure and magnetism in the layered, ferromagnetic insulator $\mathrm{CrI}_{3}$, Chem. Mater. 27, 612 (2015).

[49] B. Huang, G. Clark, E. Navarro-Moratalla, D. R. Klein, R. Cheng, K. L. Seyler, D. Zhong, E. Schmidgall, M. A. McGuire, D. H. Cobden, W. Yao, D. Xiao, P. Jarillo-Herrero, and X. Xu, Layer-dependent ferromagnetism in a van der Waals crystal down to the monolayer limit, Nature (London) 546, 270 (2017).

[50] N. D. Mermin and H. Wagner, Absence of Ferromagnetism or Antiferromagnetism in One- or Two-Dimensional Isotropic Heisenberg Models, Phys. Rev. Lett. 17, 1133 (1966).

[51] J. L. Lado and J. Fernández-Rossier, On the origin of magnetic anisotropy in two dimensional $\mathrm{CrI}_{3}, 2 \mathrm{D}$ Mater. 4, 035002 (2017).

[52] D.-H. Kim, K. Kim, K.-T. Ko, J. H. Seo, J. S. Kim, T.-H. Jang, Y. Kim, J.-Y. Kim, S.-W. Cheong, and J.-H. Park, Giant Magnetic Anisotropy Induced by Ligand $\boldsymbol{L S}$ Coupling in Layered Cr Compounds, Phys. Rev. Lett. 122, 207201 (2019). 
[53] C. Xu, J. Feng, H. Xiang, and L. Bellaiche, Interplay between Kitaev interaction and single ion anisotropy in ferromagnetic $\mathrm{CrI}_{3}$ and $\mathrm{CrGeTe}_{3}$ monolayers, npj Comput. Mater. 4, 57 (2018).

[54] I. Lee, F. G. Utermohlen, D. Weber, K. Hwang, C. Zhang, J. van Tol, J. E. Goldberger, N. Trivedi, and P. C. Hammel, Fundamental Spin Interactions Underlying the Magnetic Anisotropy in the Kitaev Ferromagnet $\mathrm{CrI}_{3}$, Phys. Rev. Lett. 124, 017201 (2020).

[55] J. G. Rau and H.-Y. Kee, Trigonal distortion in the honeycomb iridates: Proximity of zigzag and spiral phases in $\mathrm{Na}_{2} \mathrm{IrO}_{3}$, arXiv:1408.4811 [cond-mat.str-el].

[56] J. Kanamori, Electron correlation and ferromagnetism of transition metals, Prog. Theor. Phys. 30, 275 (1963).

[57] I. V. Kashin, V. V. Mazurenko, M. I. Katsnelson, and A. N. Rudenko, Orbitally-resolved ferromagnetism of monolayer $\mathrm{CrI}_{3}$, 2D Mater. 7, 025036 (2020).

[58] G. Jackeli and A. Avella, Quantum order by disorder in the Kitaev model on a triangular lattice, Phys. Rev. B 92, 184416 (2015).

[59] J. Chaloupka and G. Khaliullin, Magnetic anisotropy in the Kitaev model systems $\mathrm{Na}_{2} \mathrm{IrO}_{3}$ and $\mathrm{RuCl}_{3}$, Phys. Rev. B 94, 064435 (2016).

[60] D. R. Klein, D. MacNeill, J. L. Lado, D. Soriano, E. NavarroMoratalla, K. Watanabe, T. Taniguchi, S. Manni, P. Canfield, J. Fernández-Rossier, and P. Jarillo-Herrero, Probing magnetism in $2 \mathrm{D}$ van der Waals crystalline insulators via electron tunneling, Science 360, 1218 (2018).

[61] T. Song, X. Cai, M. W.-Y. Tu, X. Zhang, B. Huang, N. P. Wilson, K. L. Seyler, L. Zhu, T. Taniguchi, K. Watanabe, M. A. McGuire, D. H. Cobden, D. Xiao, W. Yao, and X. Xu, Giant tunneling magnetoresistance in spin-filter van der Waals heterostructures, Science 360, 1214 (2018).

[62] D. R. Klein, D. MacNeill, Q. Song, D. T. Larson, S. Fang, M. Xu, R. A. Ribeiro, P. C. Canfield, E. Kaxiras, R. Comin, and P. Jarillo-Herrero, Enhancement of interlayer exchange in an ultrathin two-dimensional magnet, Nat. Phys. 15, 1255 (2019).

[63] H. H. Kim, B. Yang, S. Li, S. Jiang, C. Jin, Z. Tao, G. Nichols, F. Sfigakis, S. Zhong, C. Li, S. Tian, D. G. Cory, G.-X. Miao, J. Shan, K. F. Mak, H. Lei, K. Sun, L. Zhao, and A. W. Tsen, Evolution of interlayer and intralayer magnetism in three atomically thin chromium trihalides, Proc. Natl. Acad. Sci. U. S. A. 116, 11131 (2019).
[64] G. Kresse and J. Hafner, Ab initio molecular dynamics for liquid metals, Phys. Rev. B 47, 558(R) (1993).

[65] J. P. Perdew, K. Burke, and M. Ernzerhof, Generalized Gradient Approximation Made Simple, Phys. Rev. Lett. 77, 3865 (1996); 78, 1396(E) (1997).

[66] A. A. Mostofi, J. R. Yates, Y.-S. Lee, I. Souza, D. Vanderbilt, and N. Marzari, wannier90: A tool for obtaining maximallylocalised Wannier functions, Comput. Phys. Commun. 178, 685 (2008).

[67] S. W. Jang, M. Y. Jeong, H. Yoon, S. Ryee, and M. J. Han, Microscopic understanding of magnetic interactions in bilayer $\mathrm{CrI}_{3}$, Phys. Rev. Mater. 3, 031001(R) (2019).

[68] N. Sivadas, S. Okamoto, X. Xu, C. J. Fennie, and D. Xiao, Stacking-dependent magnetism in bilayer $\mathrm{CrI}_{3}$, Nano Lett. 18, 7658 (2018).

[69] P. Jiang, C. Wang, D. Chen, Z. Zhong, Z. Yuan, Z.-Y. Lu, and W. $\mathrm{Ji}$, Stacking tunable interlayer magnetism in bilayer $\mathrm{CrI}_{3}$, Phys. Rev. B 99, 144401 (2019).

[70] D. Soriano, C. Cardoso, and J. Fernández-Rossier, Interplay between interlayer exchange and stacking in $\mathrm{CrI}_{3}$ bilayers, Solid State Commun. 299, 113662 (2019).

[71] L. Chen, J.-H. Chung, B. Gao, T. Chen, M. B. Stone, A. I. Kolesnikov, Q. Huang, and P. Dai, Topological Spin Excitations in Honeycomb Ferromagnet $\mathrm{CrI}_{3}$, Phys. Rev. X 8, 041028 (2018).

[72] J. Cenker, B. Huang, N. Suri, P. Thijssen, A. Miller, T. Song, T. Taniguchi, K. Watanabe, M. A. McGuire, D. Xiao, and X. Xu, Direct observation of two-dimensional magnons in atomically thin $\mathrm{CrI}_{3}$, Nat. Phys. 17, 20 (2021).

[73] P. A. Maksimov and A. L. Chernyshev, Rethinking $\alpha-\mathrm{RuCl}_{3}$, Phys. Rev. Res. 2, 033011 (2020).

[74] L. Chen, J.-H. Chung, T. Chen, C. Duan, A. Schneidewind, I. Radelytskyi, D. J. Voneshen, R. A. Ewings, M. B. Stone, A. I. Kolesnikov, B. Winn, S. Chi, R. A. Mole, D. H. Yu, B. Gao, and P. Dai, Magnetic anisotropy in ferromagnetic $\mathrm{CrI}_{3}$, Phys. Rev. B 101, 134418 (2020).

[75] J. C. Slater and G. F. Koster, Simplified LCAO Method for the Periodic Potential Problem, Phys. Rev. 94, 1498 (1954).

[76] T. Holstein and H. Primakoff, Field Dependence of the Intrinsic Domain Magnetization of a Ferromagnet, Phys. Rev. 58, 1098 (1940).

[77] J. Colpa, Diagonalization of the quadratic boson hamiltonian, Phys. A (Amsterdam) 93, 327 (1978). 